Running title: Characterization of mouse-adapted SARS-CoV-2

Keywords: Coronavirus, 2019-nCoV, SARS-CoV-2, COVID-19,

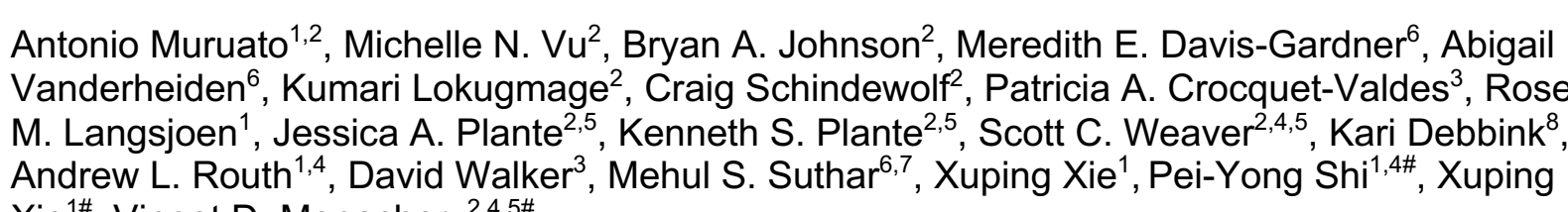

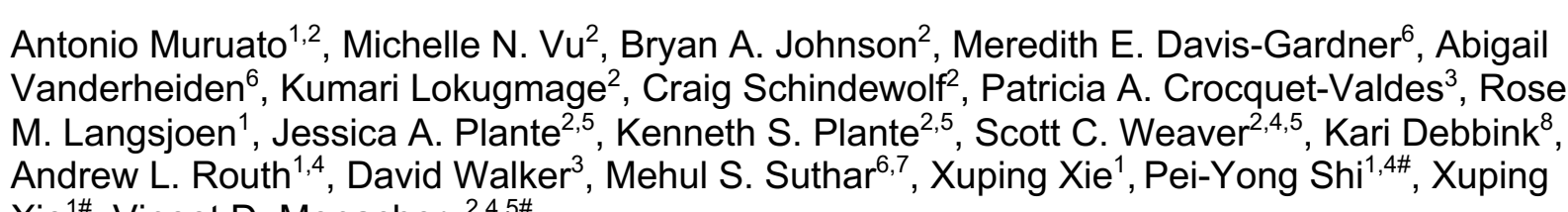

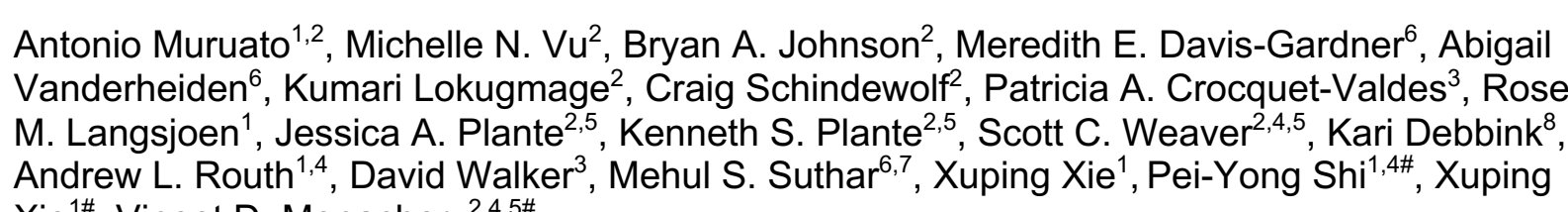

Antonio Muruato ${ }^{1,2}$, Michelle N. Vü $^{2}$, Bryan A. Johnson $\mathrm{Xie}^{1 \#}$, Vineet D. Menachery ${ }^{2,4,5 \#}$.

${ }^{1}$ Departments of Biochemistry and Molecular Biology, ${ }^{2}$ Microbiology and Immunology, ${ }^{3}$ Pathology, ${ }^{4}$ Institute for Human Infection and Immunity, ${ }^{5}$ World Reference Center of Emerging Viruses and Arboviruses, University of Texas Medical Branch, Galveston, TX, USA ${ }^{6}$ Department of Pediatrics, Emory Vaccine Center, Emory University School of Medicine, Atlanta, GA, USA.

${ }^{7}$ Yerkes National Primate Research Center, Atlanta, GA, USA

${ }^{8}$ Department of Natural Science, Bowie State University, Bowie, MD, USA

${ }^{\#}$ Co-senior authors

Corresponding Author: Vineet D. Menachery

Address: University of Texas Medical Branch, 301 University Blvd, Route \#0610 Galveston, TX 77555

Email: Vimenach@utmb.edu

\title{
Mouse Adapted SARS-CoV-2 protects animals from lethal SARS-CoV challenge
}

Co-senior authors

\section{Email: Vimenach@utmb.edu}

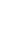




\section{Abstract}

28 The emergence of SARS-CoV-2 has resulted in a worldwide pandemic causing

29 significant damage to public health and the economy. Efforts to understand the

30 mechanisms of COVID-19 disease have been hampered by the lack of robust mouse

31 models. To overcome this barrier, we utilized a reverse genetic system to generate a

32 mouse-adapted strain of SARS-CoV-2. Incorporating key mutations found in SARSCoV-2

33 variants, this model recapitulates critical elements of human infection including

34 viral replication in the lung, immune cell infiltration, and significant in vivo disease.

35 Importantly, mouse-adaptation of SARS-CoV-2 does not impair replication in human

36 airway cells and maintains antigenicity similar to human SARS-CoV-2 strains. Utilizing this

37 model, we demonstrate that SARS-CoV-2 infected mice are protected from lethal challenge with

38 the original SARS-CoV, suggesting immunity from heterologous CoV strains. Together, the

39 results highlight the utility of this mouse model for further study of SARS-CoV-2 infection

40 and disease. 


\section{Introduction}

43 Severe acute respiratory syndrome coronavirus 2 (SARS-CoV-2), the virus that causes COVID-

4419 disease, emerged in late 2019 and has since caused an ongoing outbreak with over 153

45 million cases and over 3.2 million deaths in the last 17 months $s^{1,2}$. The novel coronavirus, similar

46 to previous emergent SARS-CoV and Middle East Respiratory Syndrome (MERS)-CoV, can

47 produce severe respiratory disease characterized by fever, labored breathing, pulmonary

48 infiltration and inflammation ${ }^{3,4}$. In severe cases, SARS-CoV-2 can lead to acute respiratory

49 distress and death. Unlike the earlier pandemic CoVs, SARS-CoV-2 maintains the ability to

50 spread asymptomatically and causes a range of disease from mild to severe ${ }^{5}$. These factors

51 have led to a world-wide outbreak that continues to rage over a year after its emergence.

In responding to the outbreak, understanding the complexity of SARS-CoV-2 infection

53 has been hampered by the limitations of small animal models ${ }^{6}$. Early on, wild-type SARS-CoV-

542 was shown to be unable to utilize mouse ACE2 for entry and infection ${ }^{7}$. Alternative models

55 utilized receptor transgenic mice expressing human ACE2 or Syrian golden hamsters to

evaluate SARS-CoV-2 infection and disease in vivo ${ }^{6}$. However, the transgenic models, while

57 causing severe disease and lethality, have distinct infection tropism leading to encephalitis in

58 addition to lung disease ${ }^{8-10}$. Similarly, while the hamster model has provided utility in studying

59 disease and transmission ${ }^{11}$, the absence of genetic knockout and immunological tools limits the

60 types of studies that can be pursued. Without a robust mouse model, many of the resources

61 used to study infection and the immune response are unavailable for SARS-CoV-2 experiments.

In order to alleviate these issues, we set out to develop a mouse-adapted strain of

63 SARS-CoV-2 using standard laboratory strains. Building from our infectious clone system ${ }^{12}$, we

64 incorporated amino acid changes that facilitated replication in standard Balb/C mice and serially

65 passaged the mutant to create a mouse-adapted strain (CMA3p20) that causes significant

66 weight loss, disease, and lung damage following infection. Notably, virus replication in this

67 model is limited to the respiratory system, thus recapitulating disease observed in most humans. 
68 Importantly, the SARS-CoV-2 CMA3p20 strain did not attenuate replication in primary human

69 airway cultures or change the antigenicity of the mouse-adapted strain relative to WT control

70 virus, making it suitable for vaccine and therapeutic studies. Finally, following prior infection with

71 SARS-CoV-2 CMA3p20, mice were protected from lethal challenge with SARS-CoV despite the

72 absence of sterilizing immunity. Together, the results highlight the utility of SARS-CoV-2

73 CMA3p20 to study infection and pathogenesis in standard mouse lines.

74 


\section{Results}

77 The initial, emergent strains of SARS-CoV-2 had spike proteins unable to utilize mouse ACE2

78 and infect standard laboratory mice ${ }^{7}$. To overcome this barrier, we generated a series of

79 mutations in the receptor-binding domain (RBD) of SARS-CoV-2 using our infectious clone ${ }^{12}$.

80 Our initial efforts modeled the interaction between SARS-CoV-2 and mouse ACE2 and used

81 previous mouse adapted strains of SARS-CoV (MA15, MA20, and v2163) ${ }^{13}$ to design mutants

82 including changes at Y449H (MA1), Y449H/L455F (MA2), and F486L/Q498Y (MA4) (S. Fig. 1A-

83 C). We also generated a series of mutants based on a reported natural SARS-CoV-2 isolate

84 (MASCP6) capable of infecting mice ${ }^{14}$, which has spike change at N501Y and several additional

85 mutations (S. Fig. 2A). Given the capacity of the MASCP6 strain to replicate in mice, we generated mutants that had the spike mutation alone (CMA1), the spike/N protein mutation (CMA2), and all four changes (CMA3) (S. Fig. 2A). For each of the six mutants, we utilized sitedirected mutagenesis in the WA1 strain clone and rescued virus stocks on Vero E6 cells (S. Fig.

2B). We subsequently infected 10 -week-old female Balb/C mice with $10^{5}$ plaque forming units (PFU) of each mutant virus and evaluated replication in the lung 2 days post infection. For WT, MA1, and MA2, no evidence of viable infection was detected in mouse lung tissues (S. Fig. 2C); however, MA4 and CMA1-3 had robust replication in mouse lung suggesting multiple combinations of RBD changes could provide compatibly with mouse ACE2 sufficient for

94 replication in a standard laboratory mouse strain.

To further evaluate the mouse adapted strains, we focused on SARS-CoV-2 CMA1, CMA2, and CMA3 mutants over a four-day time course. In female ten-week-old Balb/c mice

97 infected with $10^{5}$ PFU, none of three mutants induced major disease (S. Fig. 3A), although both

98 CMA2 and CMA3 caused more weight loss than CMA1. Examining viral replication in the lung,

99 all three mutants produced $\sim 10^{5}$ PFU per lobe at day 2 post infection (S. Fig. 3B). However, no

100 virus was detected at day 4 , suggesting rapid clearance by the host. To determine if type I

101 interferon was the major factor blunting infection, IFNAR ${ }^{-/}$SJV129 mice were infected with 
CMA1, CMA2, and CMA3 at $10^{5}$ PFU. Following infection, all three CMA mutant strains caused significant disease with both CMA2 and CMA3 peaking at $\sim 10 \%$ weight loss (S. Fig. 3 C). However, despite increased disease, viral titers were only slightly higher at day 2 than immune competent Balb/c mice and still cleared by day 4 for all three strains (S. Fig. 3D). Together, the results indicate that SARS-CoV-2 CMA1, CMA2, and CMA3 can replicate in both Balb/C and

107 IFNAR $^{-/-}$mice, but fail to sustain continued replication in vivo.

\section{8}

109

\section{Serial passage of SARS-CoV-2 CMA3}

In order to generate a SARS-CoV-2 strain that produced significant disease in an immune competent mouse, we serially passaged SARS-CoV-2 CMA3 in 10-week-old Balb/c mice. A single mouse was infected with $10^{5}$ PFU of CMA3 (p0); the mouse was subsequently euthanized at 1 day post infection with half the lung lobes taken for viral RNA and the other lobes homogenized, clarified, and used to inoculate subsequent passages (Fig. 1A); lung samples were titered by plaque assay to verify continued SARS-CoV-2 replication (Fig. 1B). After passages (p) 10, 15, and 20, stock viruses were generated on Vero E6 cells, used to infect 10-week-old Balb/C mice, and compared to the disease caused by the original CMA3 p0 strain (Fig. 1C). Following $10^{5}$ PFU challenge, mice infected with p10 and p15 were found to have augmented weight loss compared to p0; however, mice infected with p20 showed $10 \%$ weight loss by day 3 and signs of disease including ruffled fur and hunched posture. We subsequently deep sequenced the passaged virus from the lung RNA and identified two additional spike mutations (K417N and $\mathrm{H} 655 \mathrm{Y}$ ) and a mutation in the $\mathrm{E}$ protein (E8V). Several other mutations were also found as minority variants in the spike and in other parts of the genome. Modeling the receptor binding domain interaction (Fig. 1D), K417N and N501Y likely improve binding to mouse ACE2 and facilitate increased in vivo disease. Together, mouse adaptation of SARSCoV-2 CMA3 incorporated three additional fixed mutations that drive increased disease in mice.

\section{Characterization of CMA3p20.}


Having observed significant disease in mice infected with CMA3p20 relative to the initial strain of CMA3, we next evaluated weight loss, viral replication, and histopathology in Balb/c mice.

First, we tested CMA3p20 for a dose-dependent impact on weight loss (S. Fig. 4A); both $10^{6}$ and $10^{5}$ PFU caused significant, dose-dependent weight loss with minimal disease observed in the $10^{4}$ challenge. We also compared CMA3p20 infection associated weight loss to a B.1.1.7

132 SARS-CoV-2 variant (UK) which contains the N501Y mutation that permits virus replication in

133 mice (S. Fig. 3A-D). After challenge with the $10^{6}$ PFU of the B.1.1.7 variant, female Balb/C

134 mice lost approximately $10 \%$ of their starting weight by day 2 and recovered (S. Fig. 4B).

135 Together, the results indicate more severe disease with the mouse adapted CMA3p20 than the B.1.1.7 variant.

We subsequently used the $10^{5}$ PFU dose of CMA3p20 to examine infection compared to SARS-CoV-2 CMA3 over a seven-day time course. Following infection, 10-week-old female Balb/C mice CMA3p20-infected mice lost significant weight over the first four days, peaking at day 3 with $>10 \%$ weight loss (Fig. 2A). In contrast, the original CMA3 caused minimal weight

141 loss over the course of the seven-day infection. We next examined viral replication in the lung at 142 days 2, 4, and 7 post infection (Fig. 2B). CMA3p20 infection had a significant 0.5 log increase 143 in viral load over CMA3 in the lung at day 2; this difference was diminished at day 4 (0.25 log 144 increase, not statically significant) and both virus strains were cleared by day 7 in the lung. We 145 also observed day 2 replication in the trachea of mice that was cleared by day 4 in both CMA3 146 and CMA3p20 infection (Fig. 2C). Together, the data demonstrate robust weight loss and clear 147 replication in the mouse respiratory tract.

148 We next evaluated SARS-CoV-2 replication in non-respiratory tissues. Following 149 infection, we noted replication in the heart tissue of a subset of animals at day 2 (Fig. 2D).

150 However, infection was transient and not uniform in all animals and no virus was detected in the 151 heart at later time points. We subsequently evaluated viral load in the brain and blood and 152 found no evidence for CMA3 or CMA3p20 infection by plaque assay (Fig. 2E-F). To further 
153 verify viral replication, we also examined viral RNA expression in the lung, heart, brain, spleen,

154 and liver (S. Fig. 4C). While robust viral RNA was observed in the lung, the other tissues had

155 minimal evidence for CMA3p20 replication. Together, the data indicate that the SARS-CoV-2

156 CMA3p20 strain is primarily restricted to and disease driven by virus replication in the

157 respiratory tract.

158 CMA3p20 induces significant immune infiltration and lung damage.

159 Further examining lung tissue, histopathology analysis of CMA3p20 infection indicated robust

160 virus replication, immune infiltration, and tissue damage. Utilizing antigen staining against the $\mathrm{N}$

161 protein, we saw evidence for viral replication primarily in the bronchioles with additional staining

162 in the lung parenchyma at day 2 post infection (Fig. 2G). We also observed lung infiltration and

163 inflammation following CMA3p20 challenge characterized by peribronchioloitis, perivascular

164 cuffing, and perivasculitis by day 2 post infection (Fig. 2 H). Similarly, at day 4, we noted

165 collapsed airways and interstitial pneumonia (Fig. 2I). Some portions of the day 4 lungs

166 infected with CMA3p20 also had virus induced damage including enlarged and multinucleated

167 alveolar type II cells (S. Fig. 5A), loss of cellular polarity (S. Fig. 5B), and immune cells in the

168 bronchiolar lumen (S. Fig. 5D). Together, the histopathology results demonstrated significant

169 damage, inflammation, and disease in the lung following infection with SARS-CoV-2 CMA3p20.

170 CMA3p20 retains replication capacity in primary human respiratory cells.

171 Altering SARS-CoV-2 to be permissive in mice can impact its replication capacity in human cells

172 15. Therefore, we examined the ability of CMA3p20 to replicate in primary human airway

173 epithelial (HAE) cultures compared with the WT SARS-CoV-2 WA1 strain. Grown on an air

174 liquid interface, primary HAEs represent a useful in vitro model of the human airway ${ }^{16}$.

175 Following infection, CMA3p20 had equal replication to WT SARS-CoV-2 over a 72- hour time

176 course in primary HAE cultures (Fig. 3A). Similarly, viral RNA levels at 72 hours post infection

177 were equivalent between CMA3p20 and SARS-CoV-2 WA1 strain (Fig. 3B). Together, the 
results indicate that mouse adaption resulted in no significant replication attenuation of CMA3p20 in primary human airway cells.

\section{CMA3p20 retains antigenicity similar to WT SARS-CoV-2.}

181 In addition to differences in replication in human cells, spike changes in SARS-CoV-2 could alter

182 the overall antigenicity of CMA3p20 as compared to SARS-CoV-2 derived from humans; this

183 result would make it more difficult to interpret vaccine and protection studies derived from mice.

184 Therefore, to evaluate antigenicity, we infected 10 -week-old female Balb/C mice with $10^{6}$ PFU of

185 CMA3p20, then, euthanized and harvested sera 28 days post infection. We subsequently used

186 the mouse sera to measure plaque reduction neuralization titer (PRNT50) against the wild-type

187 SARS-CoV-2 WA1 strain (Fig. 3C). Mouse sera from mice $(n=7)$ infected with CMA3p20 neutralized WT SARS-CoV-2 with a PRNT50 value of $\sim 1 / 600$. To further evaluate CMA3p20 antigenicity, we examined PRNT50 assays utilizing sera from acutely infected COVID-19 patients (Fig. 3D). Performing neutralization assays in parallel, we found that CMA3p20 had $\mathrm{PRNT}^{50}$ values similar to WT SARS-CoV-2 with each COVID19 patient serum tested. With a $\mathrm{R}^{2}$ value of 0.8651 over the 13 samples, the results indicated that CMA3p20 retains similar antigenicity to the WT SARS-CoV-2 and has utility for vaccine and protection studies.

To further demonstrate the utility of CMA3p20 to understand in vivo protection, we performed a passive transfer experiment with COVID-19 patient sera. One day prior to infection, 10-week-old female BALB/C mice were pre-treated intraperitoneally with either control 197 (PBS) or 100ul of convalescent serum from a COVID-19 patient. Mice were subsequently challenged with $10^{5} \mathrm{PFU}$ of CMA3p20 and monitored for weight loss and viral titer. Mice treated 199 with acutely infected COVID-19 patient serum had significantly reduced weight loss at day 3 and

2004 post infection as compared to control mice (Fig. 3E). Similarly, viral titers in the lung were

201 reduced at both day 2 and day 4 in mice receiving COVID-19 patient serum as compared to 202 control. Consistent with the PRNT 50 data (Fig. 3D), the results from the passive transfer experiment demonstrate that antibody-based immunity generated following human infection can 
204 effectively neutralize SARS-CoV-2 CMA3p20. Together, the results confirm similar antigenicity

205 of CMA3p20 and WT SARS-CoV-2.

206 Prior SARS-CoV-2 infection protects from lethal SARS-CoV challenge.

207 Having established a SARS-CoV-2 mouse model with significant disease, we next evaluated the 208 capacity of CMA3p20 to protect against heterologous SARS-CoV challenge. Ten-week-old

209 female Balb/c mice were infected with $10^{6}$ PFU of CMA3p20 or control (PBS), monitored for 210 weight loss, and allowed to recover. CMA3p20 infected and control mice were subsequently 211 challenged with a lethal dose of mouse-adapted SARS-CoV $\left(10^{4} \mathrm{PFU}\right){ }^{17}$. Control mice infected 212 with SARS-CoV MA15 had rapid weight loss and lethality with all mice reaching euthanasia 213 criteria by day four post infection (Fig. 4A \& B). In contrast, mice previously infected with 214 SARS-CoV-2 CMA3p20 had less weight loss compared to controls, only losing approximately $21510 \%$ of their starting weight by day 2 . The CMA3p20-infected mice recovered their starting 216 weight at late times demonstrating protection from lethal SARS-CoV infection. Examining 217 disease score, mice previously infect with SARS-CoV-2 CMA3p20 showed some disease at day 2182 , but were generally devoid of ruffled fur, diminished movement, or hunching (Fig. 4C). In 219 contrast, the mock infected animals challenged with SARS-CoV showed significant disease that 220 escalated over the course of infection and required euthanasia by day 4 for all animals 221 remaining in the study $(n=10)$. For both CMA3p20 infected and uninfected animals, robust 222 SARS-CoV replication was observed in the lung (Fig. 4D). However, mice infected with 223 CMA3p20 has a significant reduction in viral loads as compared to control animals. Yet, the 224 viral replication indicates sterilizing immunity was not achieved. We subsequently evaluated the 225 neutralization capacity of SARS-CoV-2 CMA3p20 sera against SARS-CoV (Fig. 4E). CMA3p20 226 mouse sera from pre-challenge, day 2, day 4, and 7 post SARS-CoV infection were able to 227 neutralize SARS-CoV with a low range of $\mathrm{PRNT}_{50}$ values (Fig. 4F). Both the pre-challenge and 228 day 2 post challenge sera had neutralization levels $<1 / 150$, while day 4 and 7 post-challenge 229 sera were only augmented to $\sim 1 / 200$. While significantly less neutralization than what is 
bioRxiv preprint doi: https://doi.org/10.1101/2021.05.03.442357; this version posted May 4, 2021. The copyright holder for this preprint (which was not certified by peer review) is the author/funder, who has granted bioRxiv a license to display the preprint in perpetuity. It is made available under aCC-BY-NC-ND 4.0 International license.

230 observed against WT SARS-CoV-2 ( 1/600), the results suggest that SARS-CoV-2 CMA3p20

231 infection induces protection sufficient to protect from lethal challenge with SARS-CoV. 


\section{Discussion}

234 In this manuscript, we utilized a reverse genetic system ${ }^{18}$ and in vivo adaptation to generate a

235 mouse-adapted strain of SARS-CoV-2 (CMA3p20). CMA3p20 induces a dose-dependent

236 disease in young, female Balb/C mice with viral replication limited primarily to the respiratory

237 tract. In addition, CMA3p20 infection causes substantial damage to the mouse lung with

238 significant inflammation, immune infiltration, and pneumonia. Importantly, mutations in

239 CMA3p20 do not alter its ability to infect primary human cell airway cells, and the mouse

240 adapted strain maintains similar antigenicity to wild-type SARS-CoV-2. Utilizing this model, we

241 demonstrated infection with SARS-CoV-2 CMA3p20 provided protection from lethal challenge

242 with the mouse-adapted SARS-CoV. Together, results indicate the utility of CMA3p20 as a

243 model to study SARS-CoV-2 pathogenesis and immunity in standard inbred mice.

With the threat posed by SARS-CoV-2 and its emerging variants, questions have been

245 raised on the efficacy and duration of immunity following natural infection ${ }^{19,20}$. Based on the

246 protection provided by prior SARS-CoV-2 challenge against the heterologous SARS-CoV, our

247 results suggest immunity is more complex than neutralizing serum titer alone. While sera from

248 mice challenged with SARS-CoV-2 CMA3p20 neutralizes SARS-CoV at $\sim 1 / 150$, this level of

249 antibody does not provide sterilizing immunity in the lung. Even after SARS-CoV infection, the

250 serum neutralization is only $\sim 1 / 200$ at day 7 post infection. Yet, in terms of weight loss and

251 disease, the SARS-CoV-2 infected mice showed a significant reduction in severity and complete

252 protection from SARS-CoV-induced lethality.

253 From these initial findings, the exact mechanism of protection is not yet clear. With

254 previous studies with SARS-CoV, antibody-based immunity has led to complete protection from

255 viral replication in the lung ${ }^{21,22}$. Similar findings have been observed in animal models of

256 SARS-CoV-2, leading to serum neutralization as a primary correlate associated with protection

$257{ }^{23}$. However, studies with SARS-CoV have also implicated a role for cellular based immunity ${ }^{24}$.

258 Notably, initial findings have found non-neutralizing antibody responses against common cold 
259 CoV had some level of protection ${ }^{25}$. Importantly, the vast majority of T-cell studies have found 260 epitopes directed against the more conserved nucleocapsid protein ${ }^{25}$. Absent in most vaccine 261 platforms, it is unclear if the immunity stimulated by natural SARS-CoV-2 infection will mimic 262 vaccine induced immunity and offer protection against heterologous SARS-CoV challenge. In regard to the mouse adapted strain, CMA3p20, several of the key mutations in the 264 spike protein have been observed in novel SARS-CoV-2 variants of concern ${ }^{26}$. Starting with the 265 spike mutations, N501Y is represented in several COVID-19 variants of concern, found in $29 \%$ 266 of the GSAID database of reported sequences, and has been shown to improve binding to 267 human ACE2 ${ }^{27}$. In these studies, N501Y alone (CMA1) permits SARS-CoV-2 to replicate in 268 Balb/c mice. Similarly, spike mutation K417N, also found in several variants of concern, likely 269 augments receptor binding and drives in vivo disease ${ }^{28}$. In contrast to the other spike 270 mutations, $\mathrm{H} 655 \mathrm{Y}$ is outside the RBD, has low penetrance in GSAID sequences ( $<0.5 \%)$, and 271 does not have a clear functional impact ${ }^{28}$. Outside the spike changes, the other mutations 272 found in CMA3p20 are not clear in their impact. Mutations in NSP8, NSP10 (<0.5\%), and E 273 protein are rarely found in GSAID reported sequences, are not in conserved domains, and may 274 be hitchhiking mutations ${ }^{29}$. Notably, NSP6 (L37F) has been in observed in $4 \%$ of human SARS275 CoV-2 isolates, suggesting possible selection ${ }^{29}$. Similarly, while $\mathrm{N}$ change at 128 is not 276 common ${ }^{28}$, differences in disease between CMA2 and CMA1 suggesting a role in 277 pathogenesis.

While the mouse-adapted mutations in CMA3p20 render a pathogenic virus in mice, it 279 does not ablate the replication capacity in human cells or antigenicity relative to the wild-type 280 SARS-CoV-2 strains. Previously described mouse adapted strains have been shown to cause 281 significant disease but lose replication capacity in primary human airway cultures ${ }^{15}$. In these 282 studies, CMA3p20 has similar replication levels as SARS-CoV-2 WA1 in primary HAE cultures. 283 In addition, CMA3p20-infected mice generate antibody responses capable of neutralizing wild284 type SARS-CoV-2 WA1 and the mouse-adapted strain is similarly neutralized by COVID-19 
bioRxiv preprint doi: https://doi.org/10.1101/2021.05.03.442357; this version posted May 4, 2021. The copyright holder for this preprint (which was not certified by peer review) is the author/funder, who has granted bioRxiv a license to display the preprint in perpetuity. It is made available under aCC-BY-NC-ND 4.0 International license.

285 sera. Importantly, passive transfer of human convalescent sera reduced disease in mice

286 challenged with SARS-CoV-2. Together, the results indicate that CMA3p20 induces a robust

287 immune response similar to that seen in humans and useful for understanding immunity in

288 standard animal models. 
Figure 1. Mouse-adaptation of SARS-CoV-2. a) Schematic of adaptation of SARS-CoV-2 CMA3p20. One ten-week-old female Balb/c mice was infected with SARS-CoV-2 CMA3 for 1 day, euthanized, and lung tissues harvested for viral RNA and viral titer determination. Lung tissues were homogenized, clarified, and $50 \mathrm{ul}$ used to inoculate subsequent animals for 20 passages (p). b) Viral replication of CMA3 p1-p20 from lung homogenates isolated from infected

c) Stock virus generated at passages $0,10,15$, and 20 was used to infect 5 female Balb/c mice at $10^{5}$ PFU and evaluated for weight loss over a 4-day time course. stock virus. Table includes Sanger equivalent accumulation of mutations over passages p5, p10, p15, p20, and final stock used for subsequent studies. e) Modeling RBD spike mutations N501Y and $\mathrm{K} 417 \mathrm{~N}$ found in CMA3p20 with mouse ACE2.

Figure 2. SARS-CoV-2 CMA3p20 induces disease restricted to the lung. a-f) Ten-week-old observed at h) day 2 and i) day 4 post infection.

Figure 3. CMA3p20 strain maintains human replication capacity and antigenicity. a-b) Primary human airway cultures were infected with SARS-CoV-2 WT (black) or CMA3p20 (blue) at an $\mathrm{MOI}$ of 0.01 and evaluated for a) viral titer and b) viral RNA. c) Sera collected from female

311 Balb/c mice 28 days post infection with $10^{6}$ PFU of SARS-CoV-2 CMA3p20 were evaluated for 312 capacity to neutralize WT SARS-CoV-2 via PRNT50 assay. d) PRNT50 values from COVID19 313 patient sera plotted against WT virus (y-axis) versus CMA3p20 virus (x-axis). e-f) Ten-week-old 314 female Balb/c mice were treated intraperitoneally with 100ul of human COVID19 sera or control 
315 (PBS) one day prior to infection. Mice were subsequently challenged with $10^{5}$ PFU of SARS-

316 CoV-2 CMA3p20 and evaluated for e) weight loss and f) viral titer in the lung.

317 Figure 4. Prior infection with SARS-CoV-2 protects from lethal SARS-CoV challenge. a-c)

318 Ten-week-old female Balb/c mice were previously infected with $10^{6}$ PFU of SARS-CoV-2

319 CMA3p20 (blue) or mock (black), monitored for weight loss, and allowed to recover. Twenty-eight

320 days post infection, both groups were challenged with a lethal dose (10 $\mathrm{PFU})$ of mouse-adapted

321 SARS-CoV and evaluated for a) weight loss, b) lethality, and c) disease score. d) Mice were

322 subsequently euthanized at day 2,4 , and 7 and lung tissue examined for viral replication. e) Sera

323 from CMA3p20 infected and SARS-CoV challenged were evaluated for virus neutralization

324 (PRNT50) against SARS-CoV-2 (blue) or SARS-CoV over time (no rechallenge- black, day 2 red, day 4-orange, day 7 - green).

S. Figure 1. Modeling changes to mouse-adapt SARS-CoV-2. a) Key amino acid residues found in the receptor binding domain (RBD) of mouse adapted strains of SARS-CoV were aligned to SARS-CoV-2 and used to design mouse-adapted mutations ${ }^{13}$. Key interaction sites between SARS-CoV spike and ACE2 molecules highlight in red ${ }^{30}$. b-c) Modeling of key RBD residue interactions with mouse ACE2 (PDB:2AJF) comparing b) WT SARS-Cov-2 residues versus c)

331 mutations (green) predicted to improve binding.

S. Figure 2. Construction of mouse-adapted SARS-CoV-2 Mutants. a) SARS-CoV-2 genome schematic indicating location of amino acid mutations for MA1, MA2, MA4, CMA1, CMA2, and CMA3. b) Viral replication of stock viruses of MA1, MA2, MA4, and CMA1-3 grown on VeroE6 cells. c) Viral replication of MA1, MA2, MA4, and CMA1-3 from lung homogenates isolated from 336 infected mice 2 days post infection.

337 S. Figure 3. SARS-CoV-2 mutants CMA1, CMA2, and CMA3 replicate in laboratory mice. a-

338 b) Ten-week-old female Balb/c mice infected with $10^{5}$ PFU of CMA1 (red), CMA2 (green), or 339 CMA3 (black) were examined for a) weight loss and b) viral lung titer following infection at days 2 340 and 4. c-d) Ten- to twelve-week-old female IFNAR ${ }^{-/-}$SVJ129 mice infected $10^{5}$ PFU of CMA1 
341 (red), CMA2 (green), or CMA3 (black) were examined for c) weight loss and d) viral lung titer

342 following infection at days 2 and 4 . ND- non-detected.

343 S. Figure 4. In vivo characterization of SARS-CoV-2 CMA3p20. a) Examination of ten-week-

344 old female Balb/c mice infected with SARS-CoV-2 CMA3p20 at $10^{4}, 10^{5}$, and10 PFU ( $\left.n=5\right)$. b)

345 Comparison of weight loss in ten0-week old female Balb/c mice infected with $10^{6}$ PFU of SARS-

346 CoV-2 CMA3p20 (blue) or SARS-CoV-2 variant B.1.1.7 (orange). c) RT-PCR of viral RNA load

347 found in lung, heart, brain, spleen, liver, and kidney following $10^{5}$ PFU infection of SARS-CoV-2

348 CMA3p20 2- and 4-days post infection. Dotted line signifies viral RNA value derived from mock

349 infected samples.

350 S. Figure 5. SARS-CoV-2 CMA3p20 induces significant lung damage following infection.

351 a-c) CMA3p20 infected animals 2 days post infection showing a) perivascular cuffing, b)

352 perivasculitis and c) peribronchiolitis. d-f) CMA3p20 induced lung inflammation and damage 4

353 days post infection including d) cytopathic effect of the virus, e) loss of cellular polarity, and f)

354 inflammatory cells in the lumen. 
a

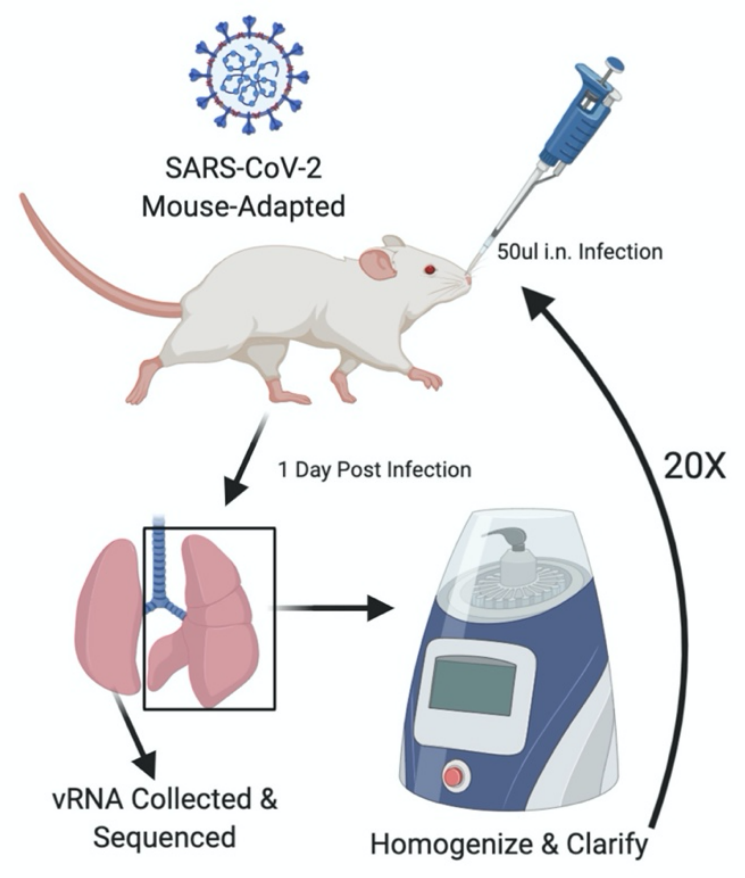

b

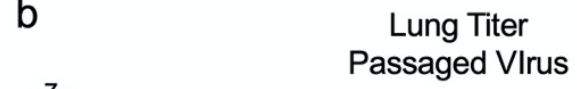

d

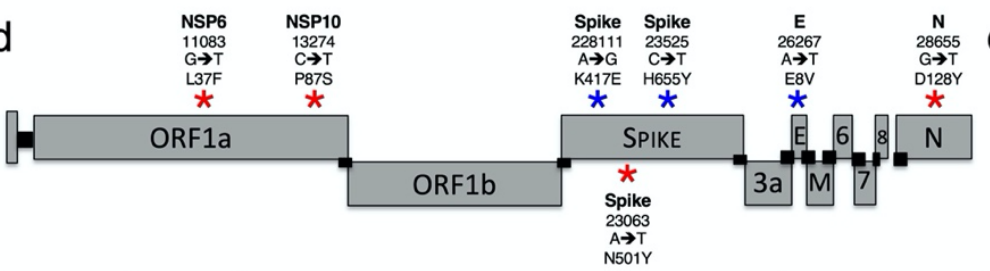

\begin{tabular}{|c|c|c|c|c|c|c|c|c|}
\hline & NSP6 & NSP10 & \multicolumn{4}{|c|}{ Spike } & E & $\mathbf{N}$ \\
\hline Nucleotide & G11083 & C13274 & A22811 & G22813 & A23063 & C23525 & A26267 & G28655 \\
\hline $\mathrm{PO}$ & $\begin{array}{c}\mathrm{T} \\
(\mathrm{L} 37 \mathrm{~F})\end{array}$ & $\begin{array}{c}\mathrm{T} \\
\text { (P87S) }\end{array}$ & & & $\begin{array}{c}\mathrm{T} \\
(\mathrm{N} 501 \mathrm{Y})\end{array}$ & & & $\begin{array}{c}\mathrm{T} \\
(\mathrm{D} 128 \mathrm{Y})\end{array}$ \\
\hline P5 & $\begin{array}{c}\mathrm{T} \\
\text { (L37F) }\end{array}$ & $\begin{array}{c}\mathrm{T} \\
\text { (P87S) }\end{array}$ & $\begin{array}{c}\mathrm{C} \\
(\mathrm{K} 417 \mathrm{Q})\end{array}$ & & $\begin{array}{c}\mathrm{T} \\
\text { (N501Y) }\end{array}$ & & & $\begin{array}{c}\mathrm{T} \\
\text { (D128Y) }\end{array}$ \\
\hline P10 & $\begin{array}{c}\mathrm{T} \\
\text { (L37F) }\end{array}$ & $\begin{array}{c}\mathrm{T} \\
\text { (P87S) }\end{array}$ & $\begin{array}{l}\text { C G mix } \\
\text { (K417E) }\end{array}$ & & $\begin{array}{c}\mathrm{T} \\
\text { (N501Y) }\end{array}$ & & & $\begin{array}{c}\mathrm{T} \\
\text { (D128Y) }\end{array}$ \\
\hline P15 & $\begin{array}{c}\mathrm{T} \\
\text { (L37F) }\end{array}$ & $\begin{array}{c}\mathrm{T} \\
\text { (P87S) }\end{array}$ & $\begin{array}{c}\mathrm{G} \\
(\mathrm{K} 417 \mathrm{E})\end{array}$ & & $\begin{array}{c}\mathrm{T} \\
(\mathrm{N} 501 \mathrm{Y})\end{array}$ & & $\begin{array}{c}\mathrm{T} \\
(\mathrm{E} 8 \mathrm{~V})\end{array}$ & $\begin{array}{c}\mathrm{T} \\
\text { (D128Y) }\end{array}$ \\
\hline P20 & $\begin{array}{c}T \\
\text { (L37F) }\end{array}$ & $\begin{array}{c}T \\
\text { (P87S) }\end{array}$ & $\begin{array}{c}\mathrm{G}^{*} \\
(\mathrm{~K} 417 \mathrm{E})\end{array}$ & $\begin{array}{c}T^{*} \\
(\mathrm{~K} 417 \mathrm{~N})\end{array}$ & $\begin{array}{c}\mathrm{T} \\
(\mathrm{N} 501 \mathrm{Y})\end{array}$ & $\begin{array}{cc}\mathrm{T} \\
(\mathrm{H} 655 \mathrm{Y})\end{array}$ & $\begin{array}{c}\mathrm{T} \\
\text { (E8V) }\end{array}$ & $\begin{array}{c}\mathrm{T} \\
\text { (D128Y) }\end{array}$ \\
\hline $\begin{array}{c}\text { CMA3P20 } \\
\text { (Stock) }\end{array}$ & $\begin{array}{c}T \\
\text { (L37F) }\end{array}$ & $\begin{array}{c}\mathrm{T} \\
\text { (P87S) }\end{array}$ & & $\begin{array}{c}\mathrm{T} \\
(\mathrm{K} 417 \mathrm{~N})\end{array}$ & $\begin{array}{c}\mathrm{T} \\
(\mathrm{N} 501 \mathrm{Y})\end{array}$ & $\begin{array}{c}\mathrm{T} \\
(\mathrm{H} 655 \mathrm{Y})\end{array}$ & $\begin{array}{c}\mathrm{T} \\
(\mathrm{E} 8 \mathrm{~V})\end{array}$ & $\begin{array}{c}\mathrm{T} \\
\text { (D128Y) }\end{array}$ \\
\hline
\end{tabular}
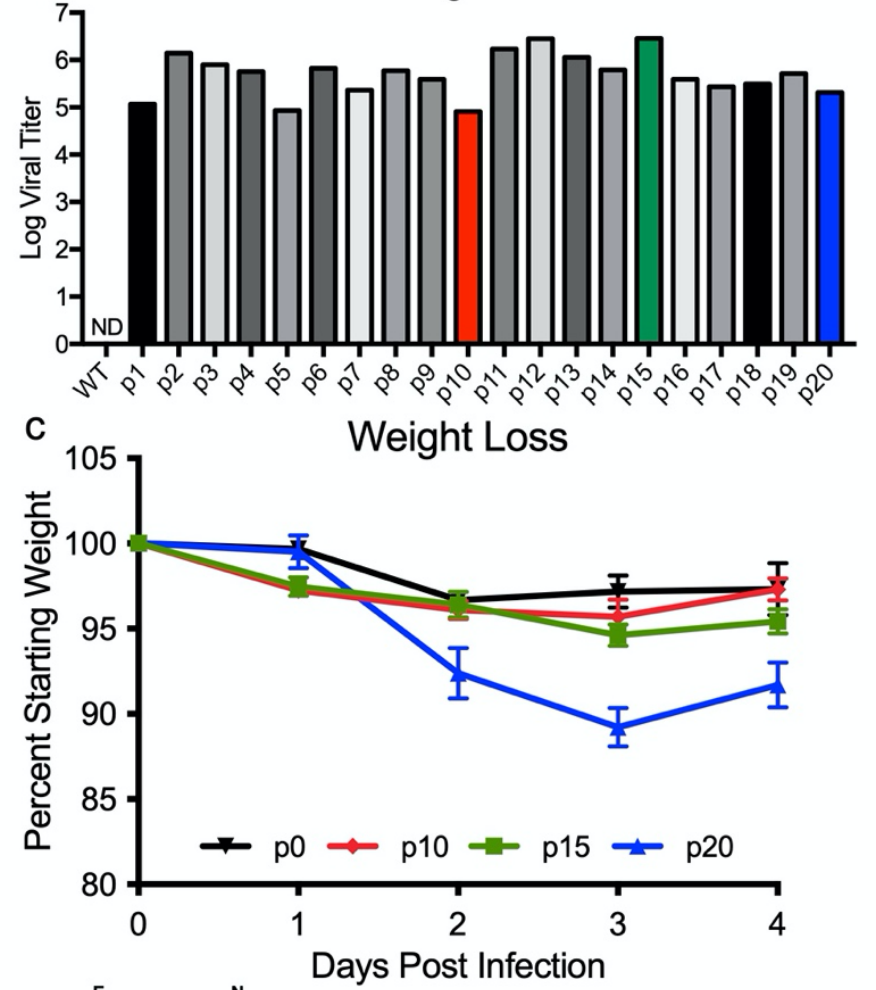

Days Post Infection

Figure 1. Mouse-adaptation of SARS-CoV-2. a) Schematic of adaptation of SARS-CoV-2 CMA3p20. One ten-week-old female Balb/c mice was infected with SARS-CoV-2 CMA3 for 1 day, euthanized, and lung tissues harvested for viral RNA and viral titer determination. Lung tissues were homogenized, clarified, and $50 \mathrm{ul}$ used to inoculate subsequent animals for 20 passages $(p)$. b) Viral replication of CMA3 p1-p20 from lung homogenates isolated from infected mice 1 day post infection. c) Stock virus generated at passages 0,10,15, and 20 was used to infect 5 female Balb/c mice at 105 PFU and evaluated for weight loss over a 4-day time course. d). Schematic of engineered (red stars) and passage-acquired (blue stars) mutations in CMA3p20 stock virus. Table includes Sanger equivalent accumulation of mutations over passages p5, p10, p15, p20, and final stock used for subsequent studies. e) Modeling RBD spike mutations N501Y and K417N found in CMA3p20 with mouse ACE2. 
a

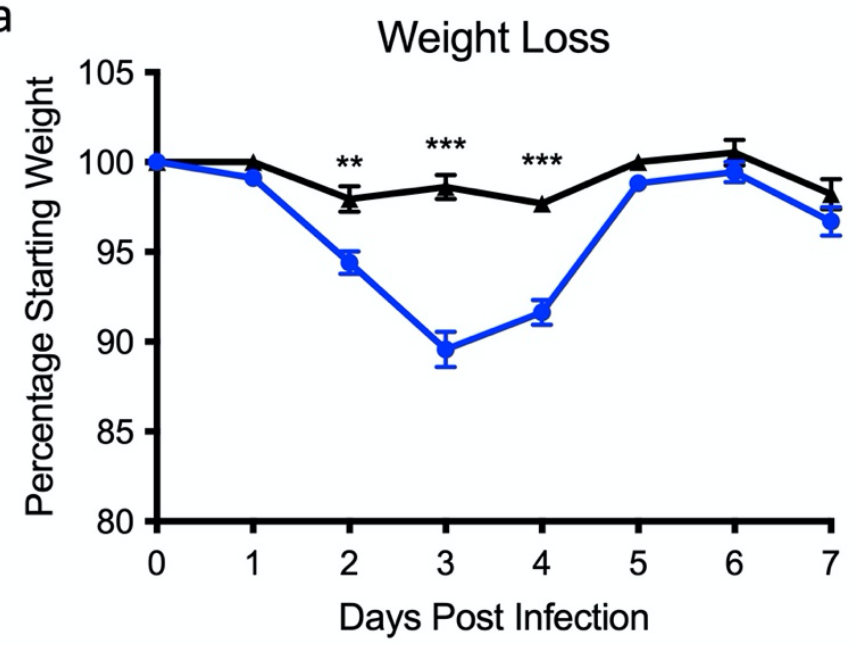

b

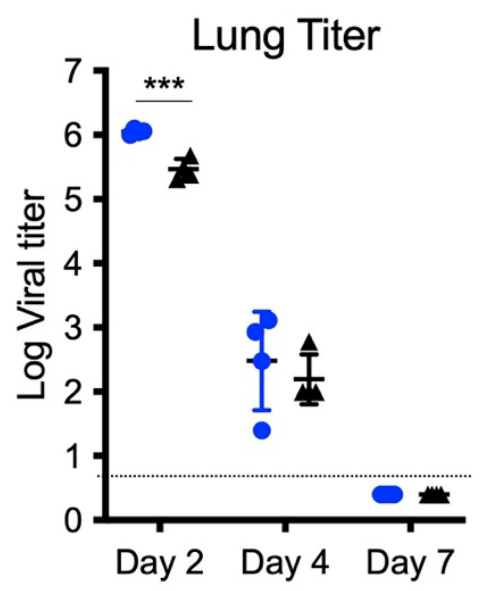

C Trachea Titer d Heart Titer e
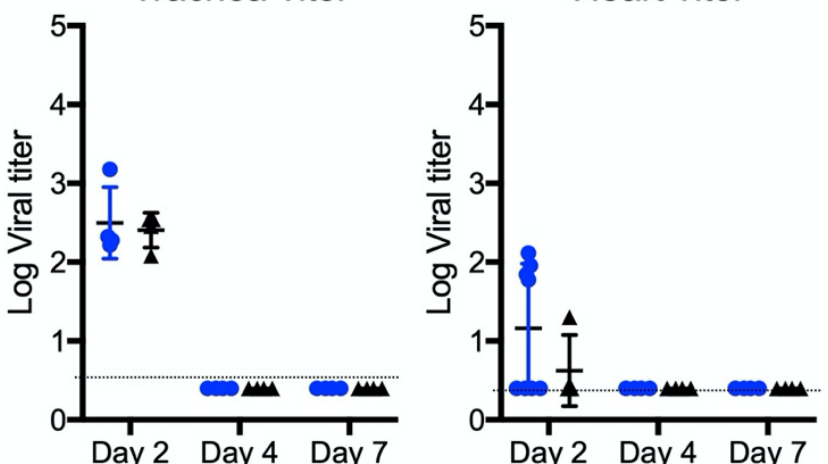

e Brain Titer
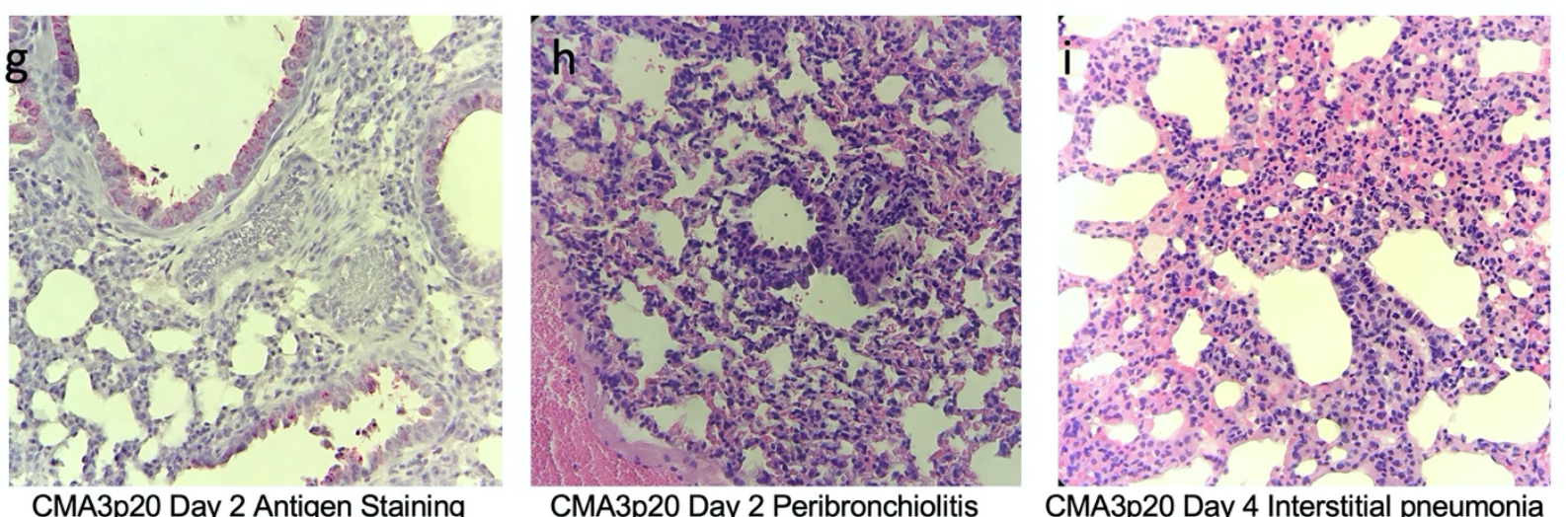

CMA3p20 Day 2 Antigen Staining

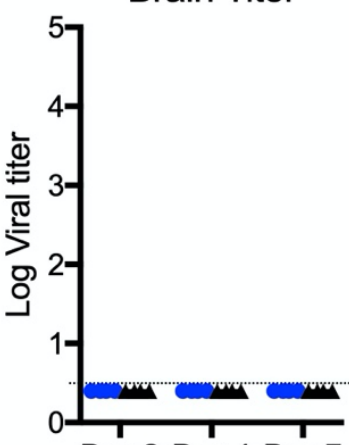

Day 2 Day 4 Day 7

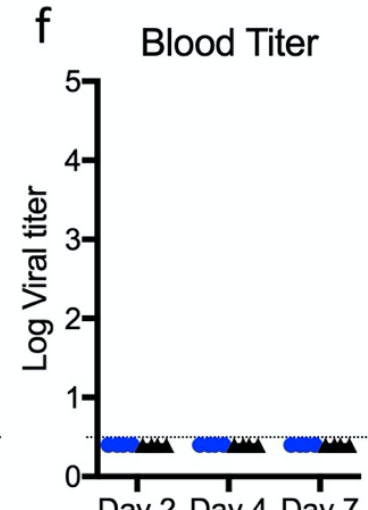

Day 2 Day 4 Day 7

Figure 2. SARS-CoV-2 CMA3p20 induces disease restricted to the lung. a-f) Ten-week-old Balb/c mice were infected with 105 PFU of SARS-CoV-2 CMA3 (black) or CMA3p20 (blue) and followed for a) weight loss and viral titer in the b) lung, c) trachea, d) heart, e) brain, and f) blood. g-i) Histology from CMA3p20 infected mice showed g) viral antigen ( $\mathrm{N}$-protein) staining in the airways and parenchyma at day 2. Significant lung infiltration, inflammation and damage was observed at h) day 2 and i) day 4 post infection. Magnification at $10 x$ for $\mathrm{g}-\mathrm{i}$. 
a

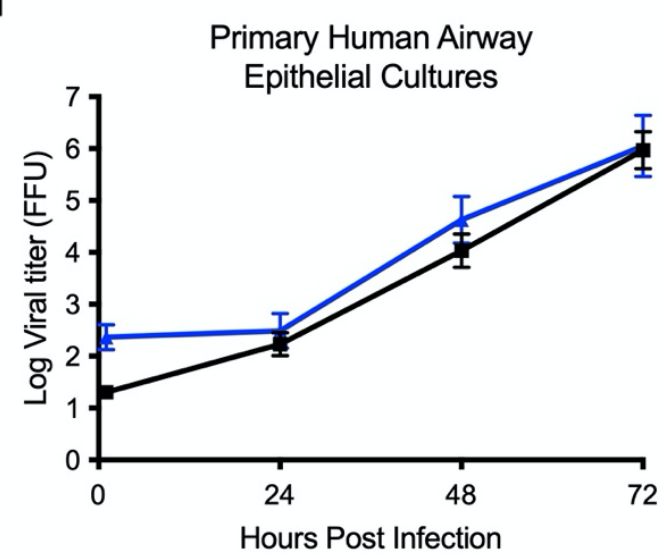

C

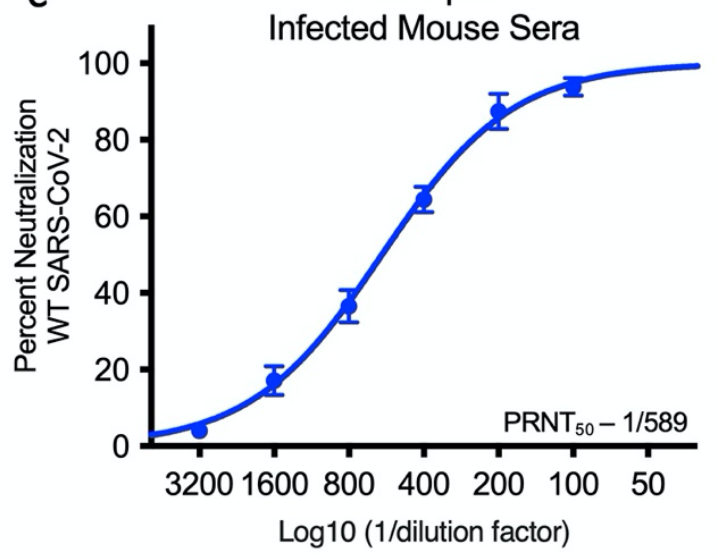

e

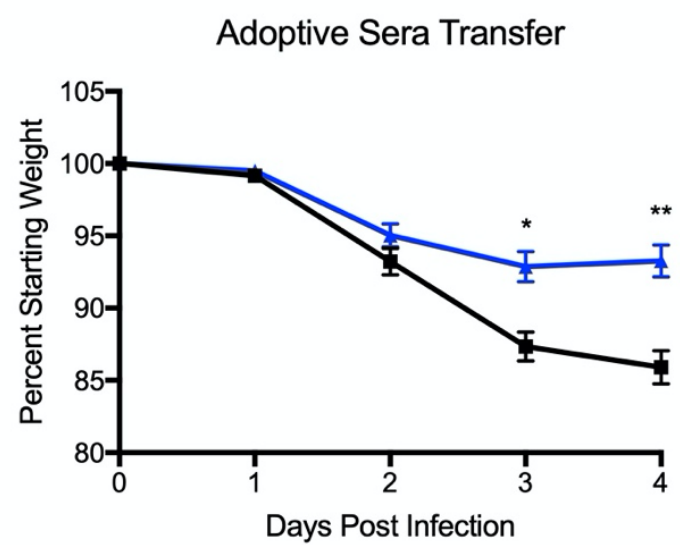

b
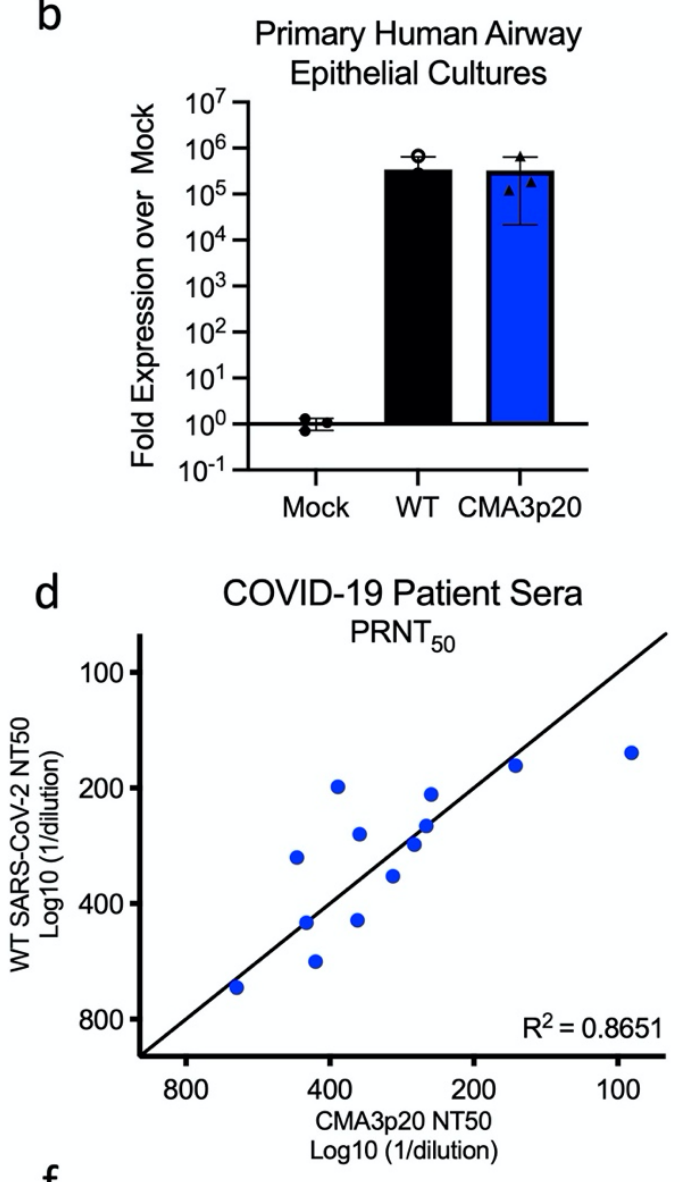

f

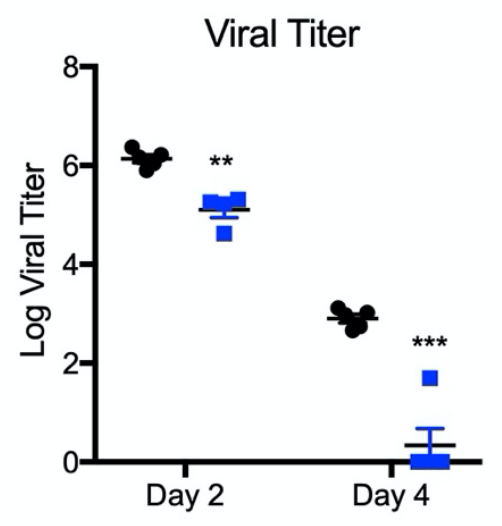

Figure 3. CMA3p20 strain maintains human replication capacity and antigenicity. a-b) Primary human airway cultures were infected with SARS-CoV-2 WT (black) or CMA3p20 (blue) at an MOI of 0.01 and evaluated for a) viral titer and b) viral RNA. c) Sera collected from female Balb/c mice 28 days post infection with 106 PFU of SARS-CoV-2 CMA3p20 were evaluated for capacity to neutralize WT SARS-CoV-2 via PRNT50 assay. d) PRNT50 values from COVID19 patient sera plotted against $W T$ virus (y-axis) versus CMA3p20 virus (x-axis). e-f) Ten-week-old female Balb/c mice were treated intraperitoneally with 100ul of human COVID19 sera or control (PBS) one day prior to infection. Mice were subsequently challenged with 105 PFU of SARS-CoV-2 CMA3p20 and evaluated for e) weight loss and f) viral titer in the lung. 
a

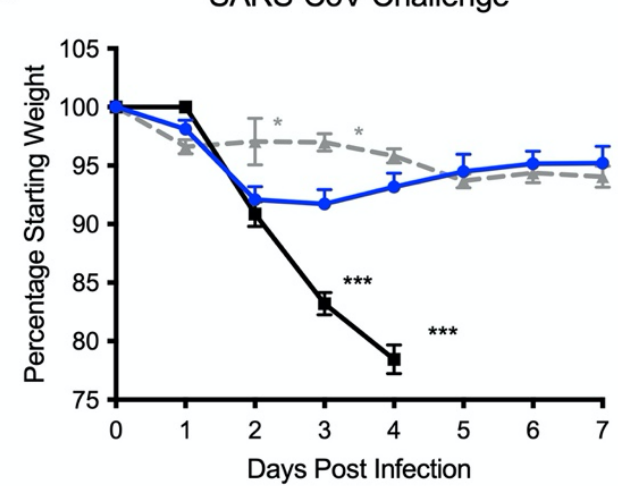

C

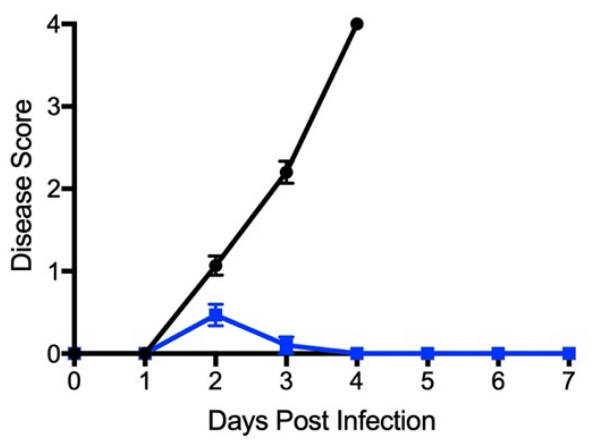

e

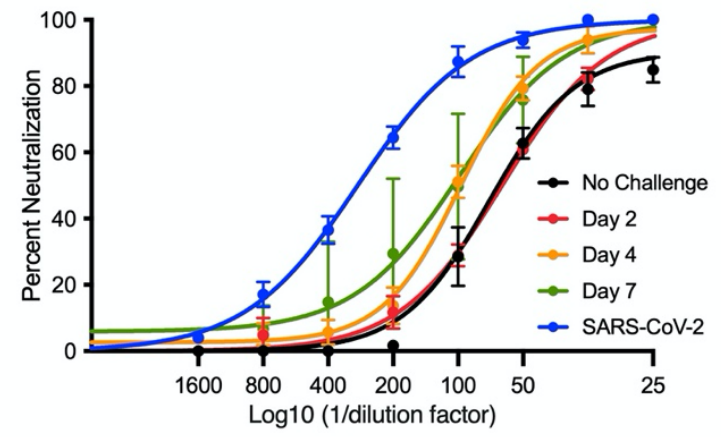

f b Survival Following

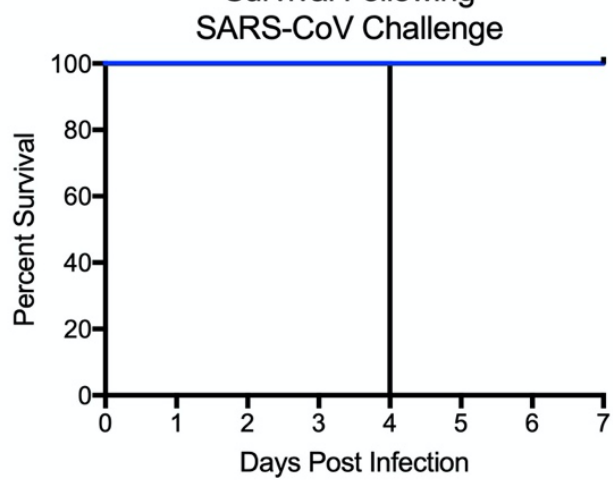

d

Lung Titer

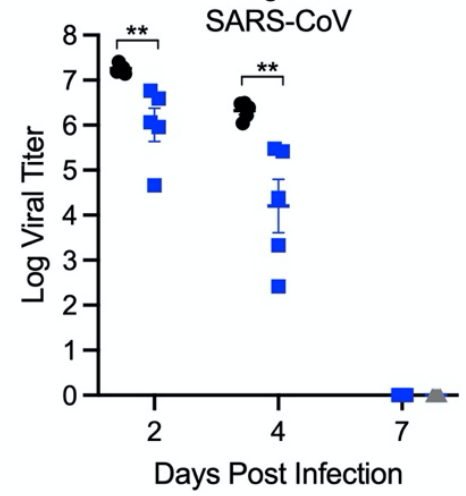

\begin{tabular}{|l|l|}
\hline Sera & PRNT $_{50}$ \\
\hline Pre-challenge & $1 / 144$ \\
\hline Day 2 & $1 / 125$ \\
\hline Day 4 & $1 / 194$ \\
\hline Day 7 & $1 / 197$ \\
\hline SARS-CoV-2 & $1 / 584$ \\
\hline
\end{tabular}

Figure 4. Prior infection with SARS-CoV-2 protects from lethal SARS-CoV challenge. a-c) CMA3p20 (blue) or mock (black), monitored for weight loss, and allowed to recover. Twentyeight days post infection, both groups were challenged with a lethal dose (104 PFU) of mouseadapted SARS-CoV and evaluated for a) weight loss, b) lethality, and c) disease score. d) Mice were subsequently euthanized at day 2, 4, and 7 and lung tissue examined for viral replication. e) Sera from CMA3p20 infected and SARS-CoV challenged were evaluated for virus neutralization (PRNT50) against SARS-CoV-2 (blue) or SARS-CoV over time (no rechallengeblack, day 2 red, day 4-orange, day 7 - green). 


\begin{tabular}{|l|c|c|c|c|c|c|c|c|c|c|c|c|c|}
\hline Strain & $\mathbf{4 0 4}$ & $\mathbf{4 2 6}$ & $\mathbf{4 3 6}$ & $\mathbf{4 4 2}$ & $\mathbf{4 7 2}$ & $\mathbf{4 7 3}$ & $\mathbf{4 7 5}$ & $\mathbf{4 7 9}$ & $\mathbf{4 8 4}$ & $\mathbf{4 8 5}$ & $\mathbf{4 8 6}$ & $\mathbf{4 8 7}$ & $\mathbf{4 9 1}$ \\
\hline SARS & $\mathrm{V}$ & $\mathrm{R}$ & $\mathrm{Y}$ & $\mathrm{Y}$ & $\mathrm{L}$ & $\mathrm{N}$ & $\mathrm{Y}$ & $\mathrm{N}$ & $\mathrm{Y}$ & $\mathrm{T}$ & $\mathrm{T}$ & $\mathrm{T}$ & $\mathrm{Y}$ \\
\hline MA15 & $\mathrm{V}$ & $\mathrm{R}$ & $\mathrm{H}$ & $\mathrm{Y}$ & $\mathrm{L}$ & $\mathrm{N}$ & $\mathrm{Y}$ & $\mathrm{N}$ & $\mathrm{Y}$ & $\mathrm{T}$ & $\mathrm{T}$ & $\mathrm{T}$ & $\mathrm{Y}$ \\
\hline MA20 & V & R & Y & L & L & N & Y & K & Y & T & T & T & Y \\
\hline MA25 & V & R & H & F & L & N & Y & N & Y & T & T & T & Y \\
\hline Strain & $\mathbf{4 1 7}$ & $\mathbf{4 3 9}$ & $\mathbf{4 4 9}$ & $\mathbf{4 5 5}$ & $\mathbf{4 8 6}$ & $\mathbf{4 8 7}$ & $\mathbf{4 8 9}$ & $\mathbf{4 9 3}$ & $\mathbf{4 9 8}$ & $\mathbf{4 9 9}$ & $\mathbf{5 0 0}$ & $\mathbf{5 0 1}$ & $\mathbf{5 0 5}$ \\
\hline SARS2 & K & N & Y & L & F & N & Y & Q & Q & P & T & N & Y \\
\hline MA1 & K & N & H & L & F & N & Y & Q & Q & P & T & N & Y \\
\hline MA2 & K & N & H & F & F & N & Y & Q & Q & P & T & N & Y \\
\hline MA4 & K & N & Y & L & L & N & Y & Q & Y & P & T & N & Y \\
\hline CMA1-3 & K & N & Y & L & F & N & Y & Q & Q & P & T & Y & Y \\
\hline
\end{tabular}

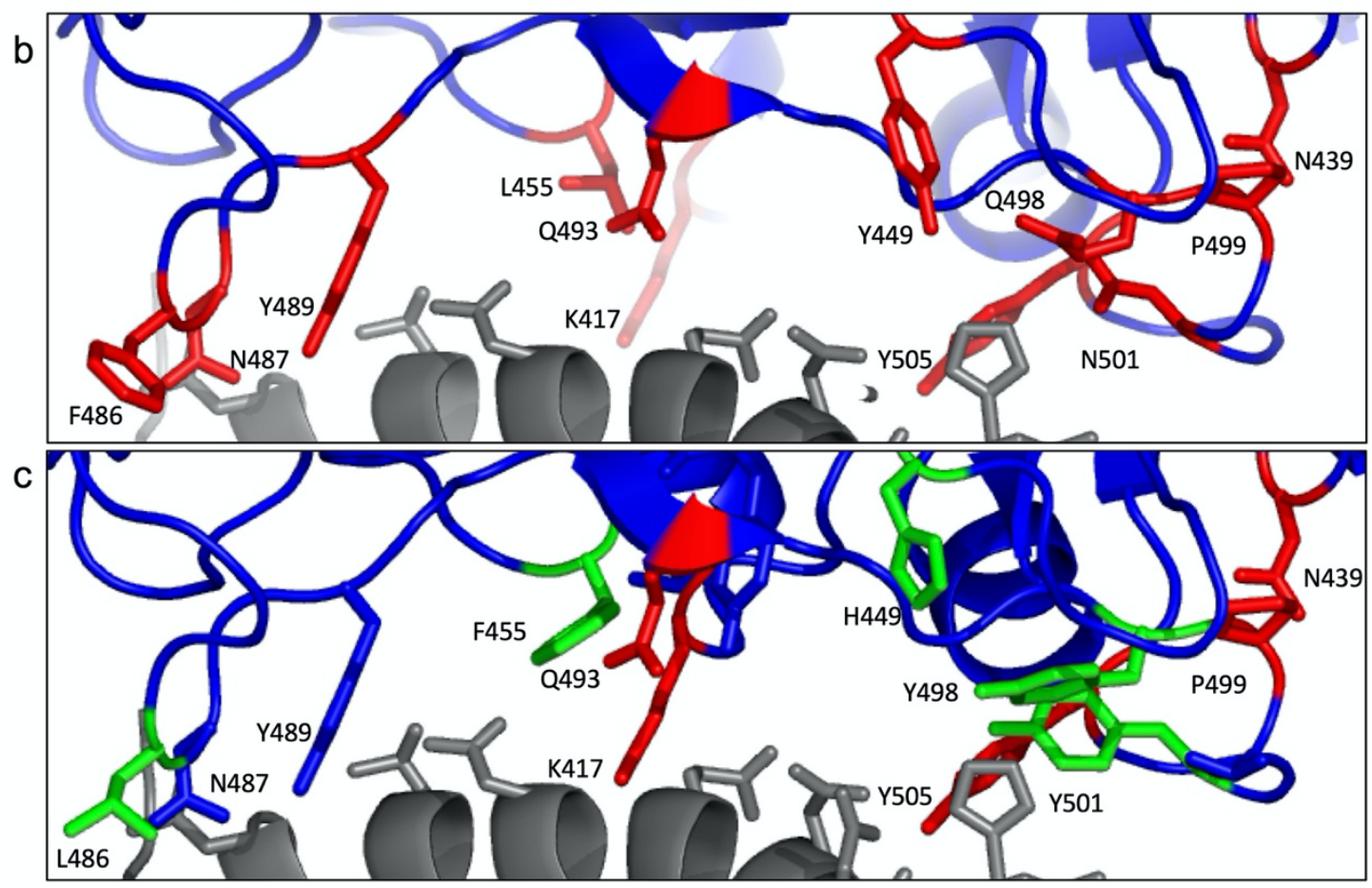

S. Figure 1. Modeling changes to mouse-adapt SARS-CoV-2. a) Key amino acid residues found in the receptor binding domain (RBD) of mouse adapted strains of SARS-CoV were aligned to SARS-CoV-2 and used to design mouse-adapted mutations 13. Key interaction sites between SARS-CoV spike and ACE2 molecules highlight in red 30. b-c) Modeling of key RBD residue interactions with mouse ACE2 (PDB:2AJF) comparing b) WT SARS-Cov-2 residues versus $c$ ) mutations (green) predicted to improve binding. 

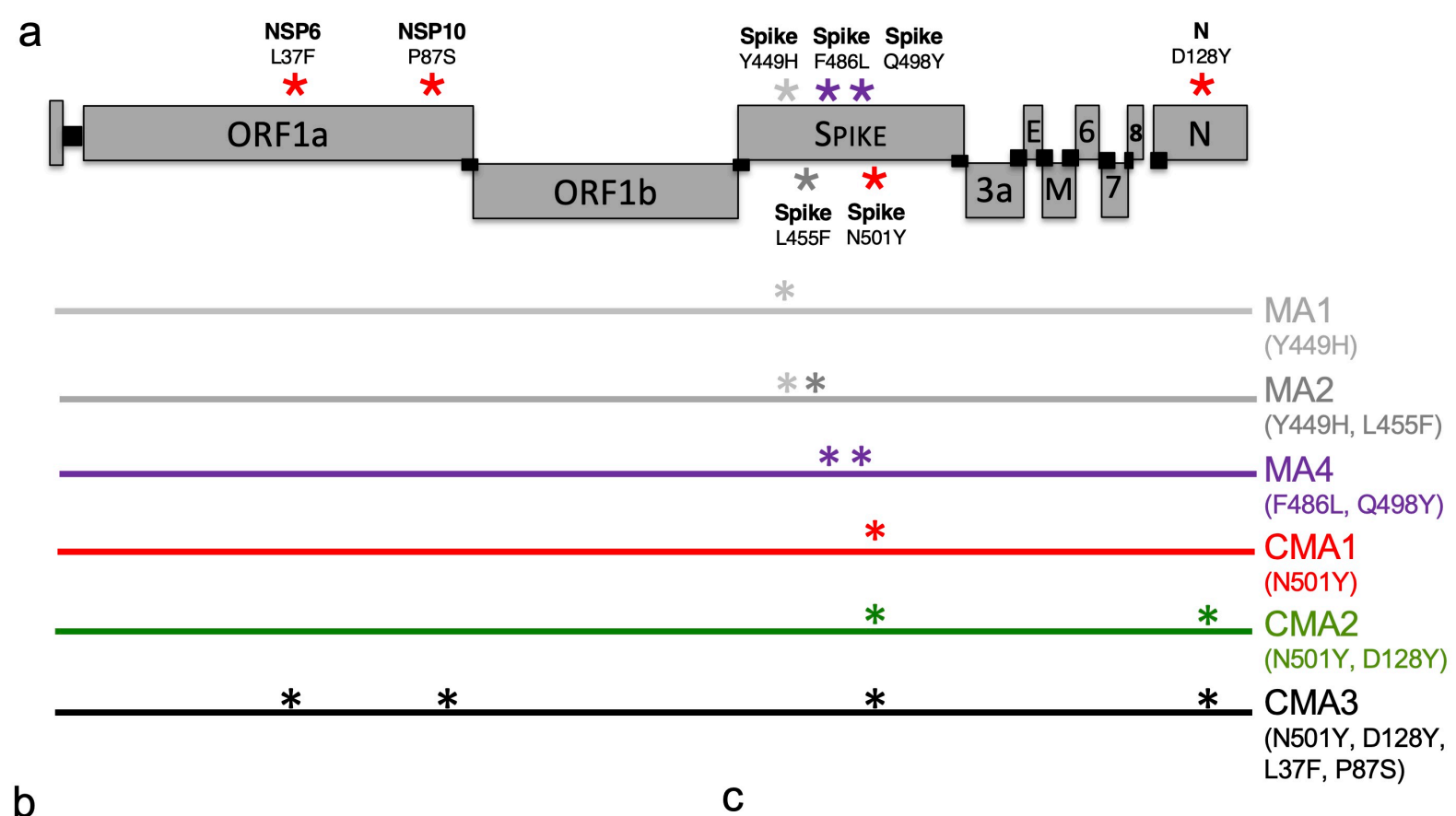

b

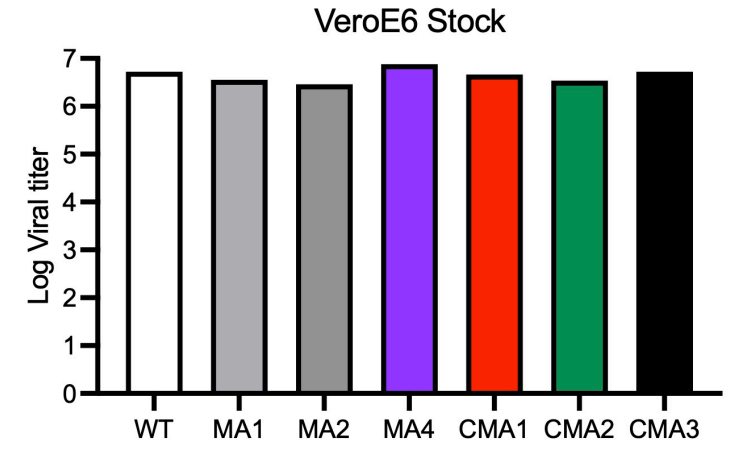

Lung Titer

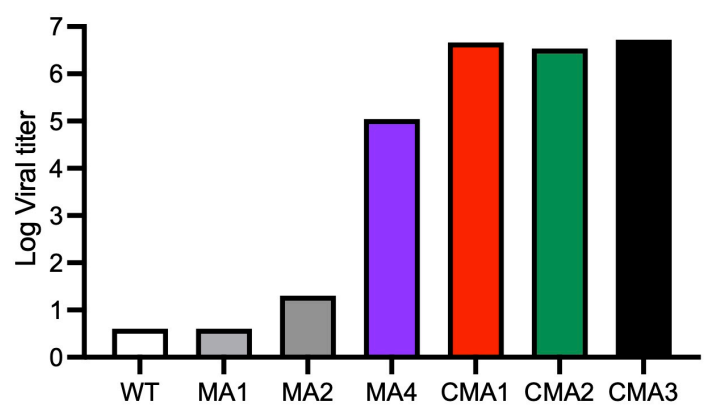

S. Figure 2. Construction of mouse-adapted SARS-CoV-2 Mutants. a) SARS-CoV-2 genome schematic indicating location of amino acid mutations for MA1, MA2, MA4, CMA1, CMA2, and CMA3. b) Viral replication of stock viruses of MA1, MA2, MA4, and CMA1-3 grown on VeroE6 cells. c) Viral replication of MA1, MA2, MA4, and CMA1-3 from lung homogenates isolated from infected mice 2 days post infection. 


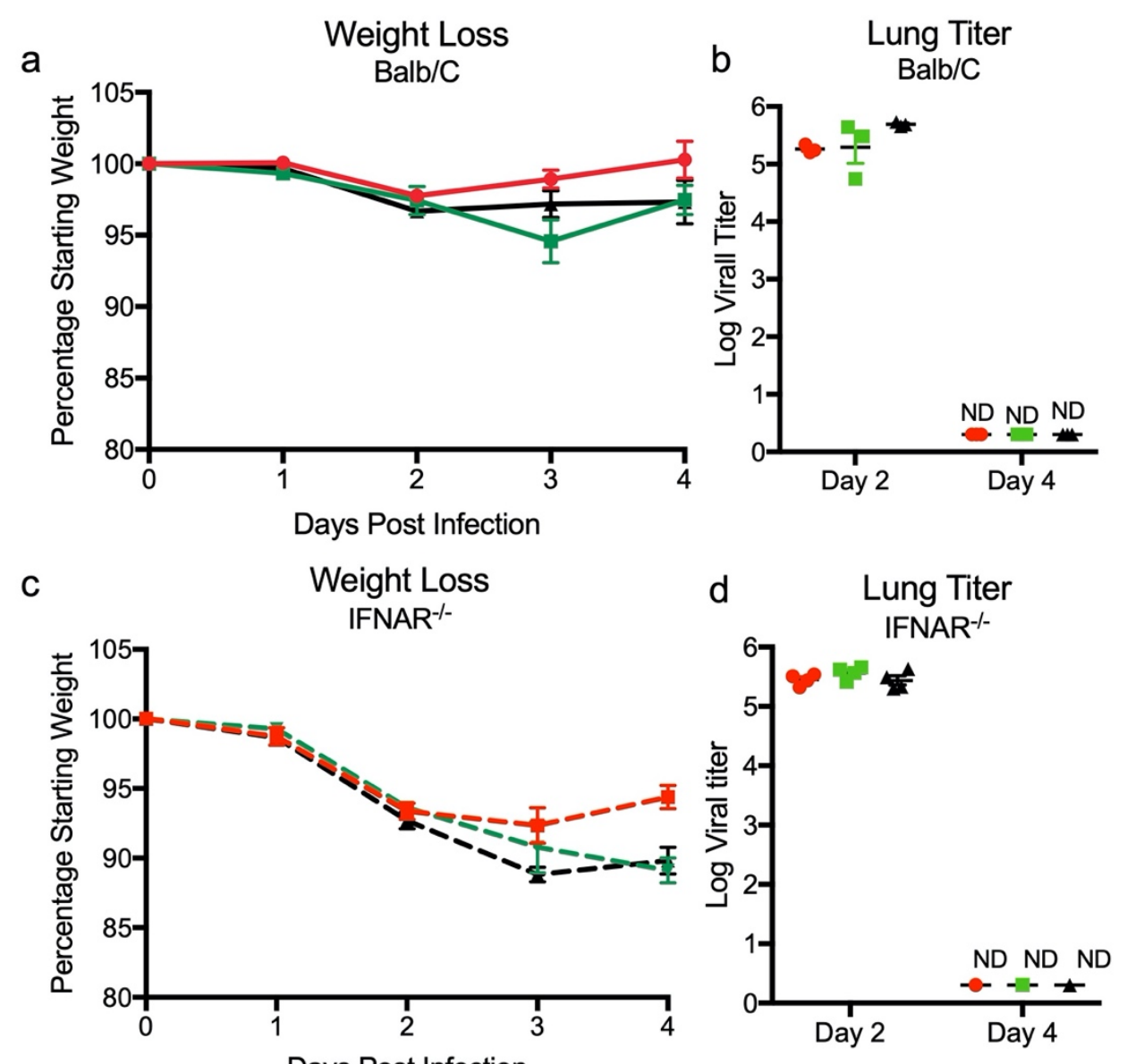

Days Post Infection

S. Figure 3. SARS-CoV-2 mutants CMA1, CMA2, and CMA3 replicate in laboratory mice. ab) Ten-week-old female Balb/c mice infected with 105 PFU of CMA1 (red), CMA2 (green), or CMA3 (black) were examined for a) weight loss and b) viral lung titer following infection at days 2 and 4. C-d) Ten- to twelve-week-old female IFNAR-/- SVJ129 mice infected 105 PFU of CMA1 (red), CMA2 (green), or CMA3 (black) were examined for c) weight loss and d) viral lung titer following infection at days 2 and 4 . ND- non-detected. 
a

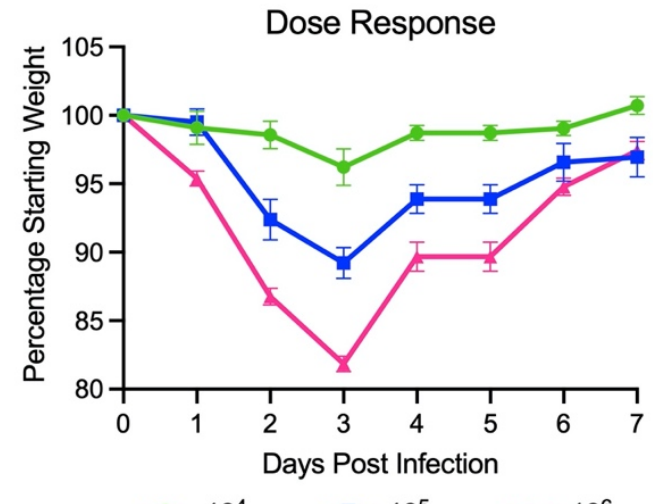

$\rightarrow 10^{4} \rightarrow 10^{5} \quad-10^{6}$ b

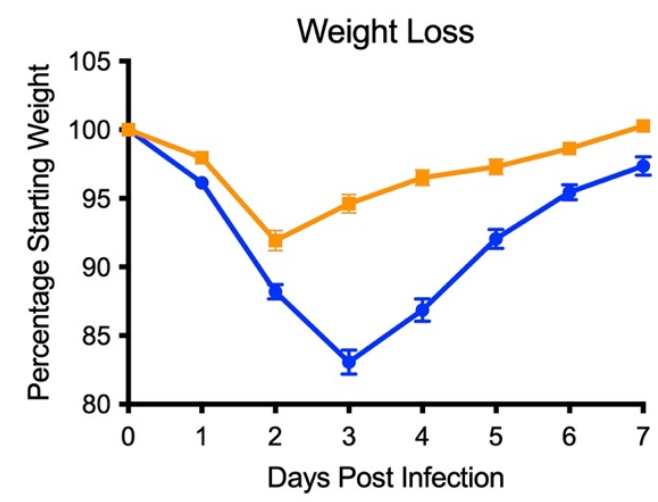

$\rightarrow$ UK Variant $\rightarrow$ CMA3p20

C

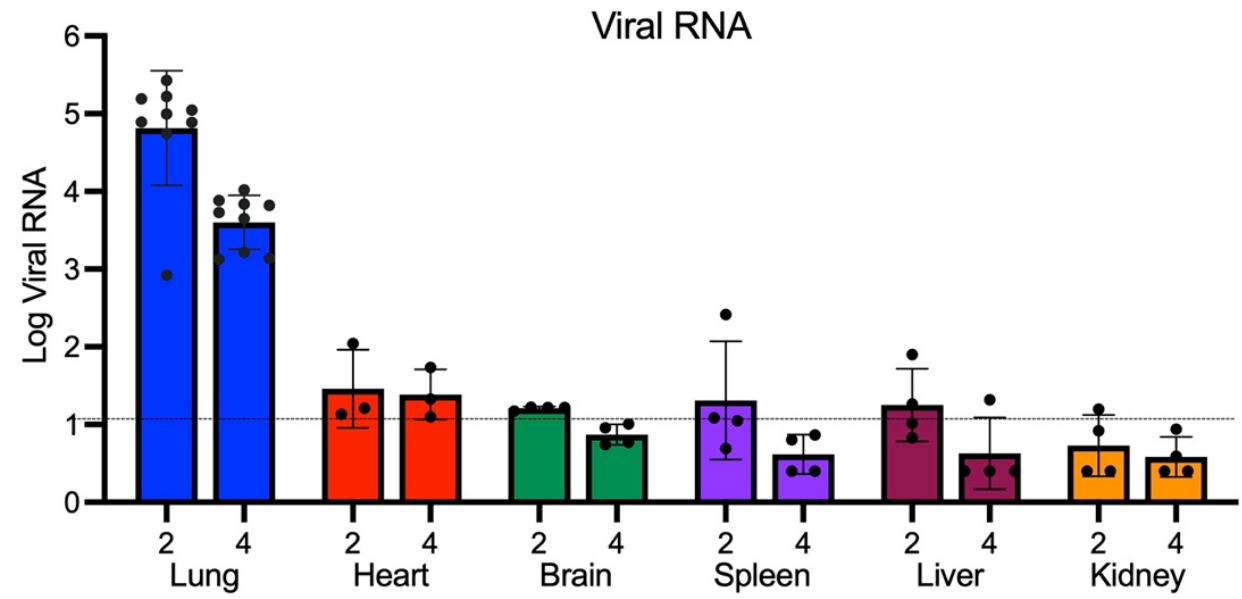

S. Figure 4. In vivo characterization of SARS-CoV-2 CMA3p20. a) Examination of ten-week-old female Balb/c mice infected with SARS-CoV-2 CMA3p20 at 104, 105, and106 PFU ( $n=5)$. b) Comparison of weight loss in ten0-week old female Balb/c mice infected with 106 PFU of SARS-CoV-2 CMA3p20 (blue) or SARS-CoV-2 variant B.1.1.7 (orange). c) RT-PCR of viral RNA load found in lung, heart, brain, spleen, liver, and kidney following 105 PFU infection of SARS-CoV-2 CMA3p20 2- and 4-days post infection. Dotted line signifies viral RNA value derived from mock infected samples. 


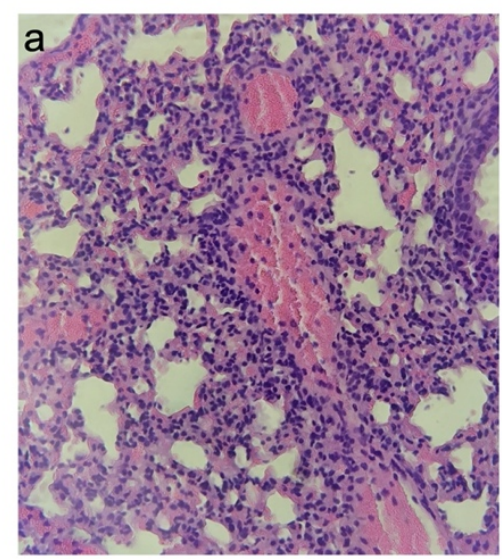

CMA3p20 Day 2 Vascular Cuffing

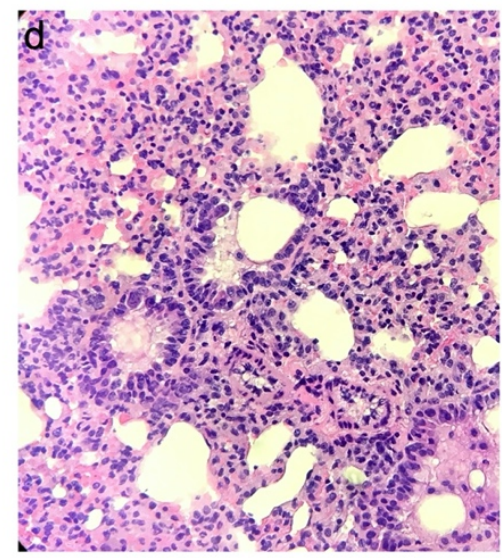

CMA3p20 Day 4 Cytopathic Effect

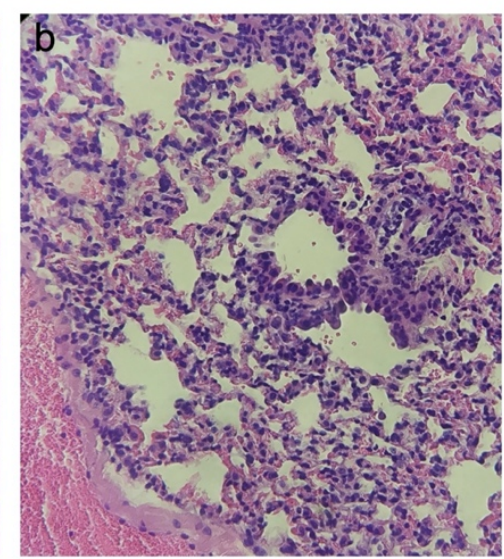

CMA3p20 Day 2 Perivasculitis

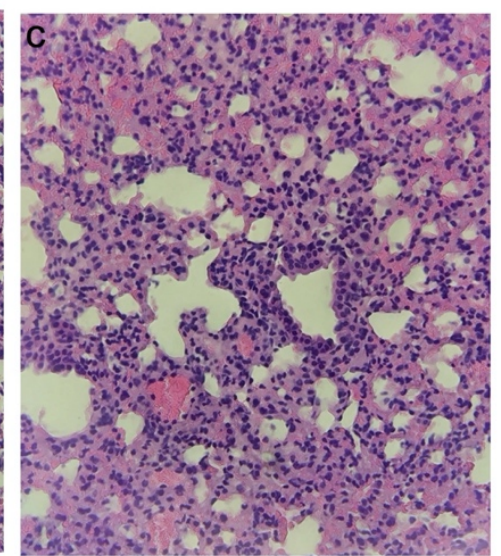

CMA3p20 Day 2 Peribronchiolitis

S. Figure 5. SARS-CoV-2 CMA3p20 induces significant lung damage following infection.

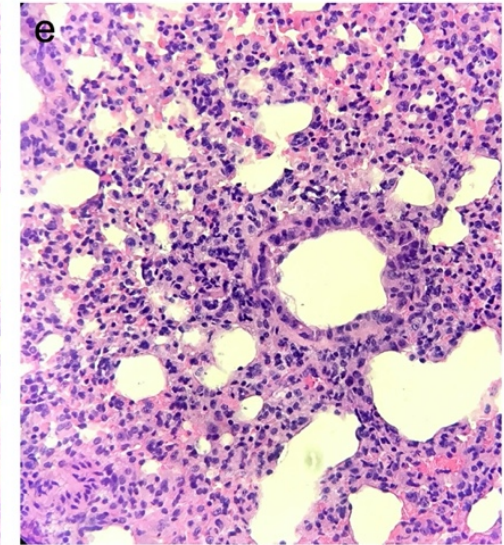

CMA3p20 Day 4 Loss of Polarity

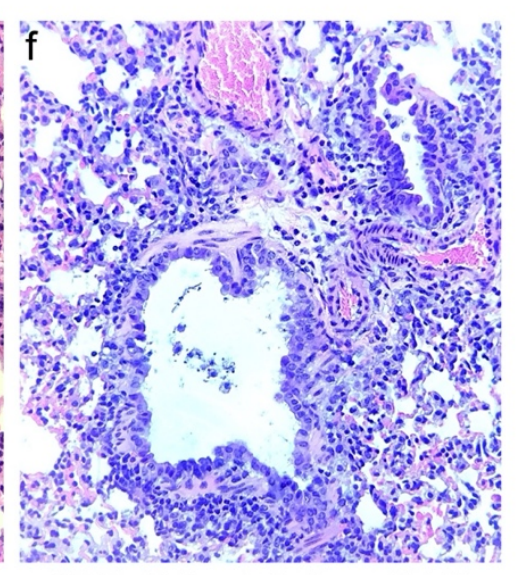

CMA3p20 Day 4 Peribronchioloitis a-c) CMA3p20 infected animals 2 days post infection showing a) perivascular cuffing, b) perivasculitis and c) peribronchiolitis. d-f) CMA3p20 induced lung inflammation and damage 4 days post infection including d) cytopathic effect of the virus, e) loss of cellular polarity, and f) inflammatory cells in the lumen. Magnification at 10x for a-f. 


\section{Methods}

430 Viruses and cells. The recombinant wild-type and mouse-adapted strains of SARS-CoV-2 are

431 based on the sequence of USA-WA1/2020 isolate provided by the World Reference Center for

432 Emerging Viruses and Arboviruses (WRCEVA) and was originally obtained from the USA Centers

433 for Disease Control and Prevention as described ${ }^{31}$. Wild-type and mutant SARS-CoV-2 as well

434 as recombinant mouse-adapted recombinant SARS-CoV ${ }^{17}$ were titrated and propagated on Vero

435 E6 cells, grown in DMEM with 5\% fetal bovine serum and 1\% antibiotic/antimycotic (Gibco).

436 Standard plaque assays were used for SARS-CoV and SARS-CoV-2 ${ }^{32,33}$. All experiments

437 involving infectious virus were conducted at the University of Texas Medical Branch (Galveston,

$438 \mathrm{TX}$ ) or Emory University (Atlanta, Georgia) in approved biosafety level 3 (BSL) laboratories with

439 routine medical monitoring of staff.

440 Phylogenetic tree, sequence identity heat map, and structural modeling. Spike receptor

441 binding tables were constructed from a set of representative group 2B coronaviruses by using

442 alignment data paired with neighbor-joining phylogenetic trees built in Geneious (v.9.1.5) using

443 the spike amino acid sequences derived the following accession numbers: QHU79204 (SARS-

444 CoV-2 WA1), AGZ48806 (RsSHC014), ALK02457 (WIV16), and AYV99817.1(SARS-CoV

445 Urbani). Mouse-adapted SARS-CoV-2 structural homology models were generated using 446 SWISS-Model ${ }^{34,35}$ with the 6LZG crystal structure (RCSB Protein Data Bank) as the template 447 structure for the spike protein and the 2AJF crystal structure (RCSB Protein Data Bank) as the 448 template for ACE2. Homology models were visualized and manipulated in PyMOL (version 2.4.2).

449 Construction of mouse-adapted mutant SARS-CoV-2. Both wild-type and mutant viruses were 450 derived from the SARS-CoV-2 USA-WA1/2020 infectious clone as previously described ${ }^{12}$. For 451 mouse adapted virus construction, the individual mutations were synthesized and introduced into 452 the appropriate plasmids (F1-F7) via PCR-based mutagenesis with synthesized specific primers 453 containing corresponding mutations. The resulted plasmid was validated by further restriction 
enzyme digestion and Sanger sequencing. Thereafter, plasmids containing wild-type and mutant SARS-CoV-2 genome fragments were amplified and digested by restriction enzyme. The SARSCoV-2 genome fragments were purified and ligated in vitro to assemble the full-length cDNA 457 according to the procedures described previously ${ }^{12,36}$. In vitro transcription reactions then were 458 preformed to synthesize full-length genomic RNA. To recover the viruses, the RNA transcripts 459 were electroporated into Vero E6 cells. The medium from electroporated cells as harvested at 40 460 hours post transfection and served as seed stocks for amplifying one passage on Vero E6 cells 461 (P1 stock). Viral mutants were confirmed by sequence analysis prior to use. Synthetic 462 construction of SARS-CoV-2 mouse adapted strains were approved by the University of Texas 463 Medical Branch Institutional Biosafety Committee.

464 In vitro infection. Viral infections in primary human airway cells were performed as previously 465 described ${ }^{37}$. Briefly, the apical side of the HAE cultures were washed 3 times with PBS. Cultures were infected with SARS-CoV-2 WT (WA1) or CMA3p20 at MOI 0.01 and allowed to adsorb for

$4671 \mathrm{hr}$ at $37^{\circ} \mathrm{C}$. After adsorption, the apical side was washed 3 times with PBS, and the basolateral 468 media replaced. Viral washes were collected adding PBS to the apical side and incubated for 30 469 minutes at $37^{\circ} \mathrm{C}$. Viral titer was evaluated by focus forming assay as previously described ${ }^{37}$. 470 RNA from HAE were collected at 48 hours post infection. RNA was harvested from mock-infected 471 and infected HAE cultures by treating with RNA lysis buffer for $>5$ min and gently pipetting to 472 recover cells. Total RNA was extracted using the Zymo Quick-RNA miniprep kit (VWR; R1055) 473 according to the manufacturer's protocol. Purified RNA was reverse transcribed into cDNA using 474 the high-capacity cDNA reverse transcription kit (Thermo Fisher, 43-688-13). RNA levels were 475 quantified using the IDT Prime Time gene expression master mix and TaqMan gene expression 476 Primer/Probe sets (IDT) and run on a QuantStudio5 qPCR system using SARS-CoV-2 RDRP477 specific primers (forward [F], GTGARATGGTCATGTGTGGCGG; reverse [R], 478 CARATGTTAAASACACTATTAGCATA) and probe (56-6-carboxyfluorescein 
[FAM]/CAGGTGGAA/ZEN/CCTCATCAGGAGATGC/3IABkFQ) were used. Three or more

480 biological replicates were harvested at each described time and results are representative of

481 multiple experiments. No blinding was used in any sample collections, nor were samples

482 randomized. Microsoft Excel for Mac 2011 was used to analyze data.

483 Deep sequencing analysis. RNA libraries of SARS-CoV-2 mutants were prepared with $300 \mathrm{ng}$

484 of RNA using the Tiled-ClickSeq protocol as previously described ${ }^{38,39}$ using tiled primers cognate

485 to the SARS-CoV-2 genome (accession number NC_045512.2) and the TruSeq i7 LT adapter

486 series and i5 hexamer adaptors containing a 12N unique molecular identifier (UMI). Libraries were

487 sequenced on the Illumina MiSeq platform with MiSeq Reagent Kit v2 using paired-end reads

488 (R1:250 cycles, R2:50 cycles). Raw data was de-multiplexed using TruSeq indexes using the

489 MiSeq Reporter Software. Demultiplexed read data were quality filtered, adaptor-trimmed and

490 primer-trimmed as previously described ${ }^{39}$. Reads were mapped to the WA-1 reference

491 (NC_045512.2) using ViReMa ${ }^{40}$. Reads were de-duplicated with umi_tools ${ }^{41}$ using $12 \mathrm{~N}$ unique

492 molecular identifiers (UMIs) embedded in the i5 click-adaptor. A consensus reference sequence

493 was generated using Pilon ${ }^{42}$ ensuring that read coverage was greater than $25 x$ across $99.5 \%$ of

494 the reference genome. Pileup files were generated using Samtools v1.9 ${ }^{43}$ and minority variants

495 were extracted by nucleotide voting (PHRED>=30) using a custom python3 script previously

496 described ${ }^{39}$.

497 Plaque reduction neutralization test. Neutralization assays were preformed using conventional 498 plaque reduction neutralization assay (PRNT50) as previously described ${ }^{44}$. Briefly, 100 PFU of 499 SARS-CoV-2, mouse-adapted SARS-CoV-2, or SARS-CoV MA15 was incubated with serially 500 diluted serum from mice or COVID-19 patients (total volume of $200 \mu \mathrm{l}$ ) at $37^{\circ} \mathrm{C}$ for $1 \mathrm{~h}$. The virus501 serum mixture was added to the pre-seeded Vero E6 cells. After $1 \mathrm{~h} 37^{\circ} \mathrm{C}$ incubation, $2 \mathrm{ml}$ of $2 \%$ 502 high gel temperature agar (SeaKem) in DMEM containing 5\% FBS and 1\% P/S was overlaid onto 503 infected cells. After 2 days of incubation, $2 \mathrm{ml}$ neutral red ( $1 \mathrm{~g} / \mathrm{l}$ in PBS; Sigma) was added to the 
504 agar-covered cells. After another 5-h incubation, neutral red was removed. Plaques were counted

505 for NT50 calculation. The PRNT assay was performed at the BSL-3 facility at UTMB.

506 Ethic Statement. This study was carried out in accordance with the recommendations for care 507 and use of animals by the Office of Laboratory Animal Welfare, National Institutes of Health. The 508 Institutional Animal Care and Use Committee (IACUC) of University of Texas Medical Branch

509 (UTMB) approved the animal studies under protocol 1711065 and 1707046 . For samples Emory

510 University, collection and processing were performed under approval from the University

511 Institutional Review Board (IRB \#00001080 and \#00022371). Adults $\geq 18$ years were enrolled who

512 met eligibility criteria for SARS-CoV-2 infection (PCR or rapid antigen test confirmed by a

513 commercially available assay) and provided informed consent.

\section{Human Serum samples}

515 For Emory University, acute peripheral blood samples were collected from hospitalized patients 516 at the time of enrollment. Infected patients were randomly selected from a convenience sample

517 and no data was collected on the number of patients that were pre-screened or declined 518 participation. All patients enrolled in July 2020 and had a mean age of 57 (range: 26-85; 50\% 519 male). Samples were collected in the first 9 days (range: 2-9) of their hospital stay (range: 3-33 520 days) and mostly 1-2 weeks after symptom onset (range 5-19 days), the majority of the patients 521 had comorbid conditions $(n=16)$ with 19 out of 20 having severe disease and one patient had 522 moderate disease. All of these patients had radiological evidence of pneumonia; 19 out of the 20 523 patients required supplemental oxygen, and 4 out of 20 patients were admitted to the intensive 524 care unit (ICU). Three enrolled patients died of COVID-19.

525 Mice and in vivo infection. Ten-week-old BALB/C mice were purchased from Charles River 526 Laboratories and were maintained in Sealsafe ${ }^{T M}$ HEPA-filtered air in/out units. Prior to infection, 527 animals were anesthetized with isoflurane and infected intranasally (IN) with $10^{4}$ to $10^{6}$ plaque 528 forming units diluted $50 \mu$ of phosphate-buffered saline (PBS). Infected animals were monitored 529 for weight loss, morbidity, and clinical signs of disease, and lung titers were determined as 
530 described previously ${ }^{45}$. Infected animals were weighed daily, and lung tissue collected 2-, 4- and

531 7-days post infection for downstream analysis by plaque assay.

532 Real-Time PCR for Viral RNA. RNA from tissues were collected using RNA later (). Samples

533 were subsequently homogenized with Trizol reagent (Invitrogen). RNA was then extracted from

534 Triazol using the Direct-zol RNA Miniprep Plus kit (Zymo Research \#R2072) per the

535 manufacturer's instruction. Extracted RNA was then converted to cDNA with the iScript cDNA

536 Synthesis kit (BioRad \#1708891). Quantitative real time PCR (qRT-PCR) was performed with the

537 Luna Universal qPCR Master Mix (New England Biolabs \#M3003) on a CFX Connect instrument

538 (BioRad \#1855200). Primer 1 (Forward - AAT GTT TTT CAA ACA CGT GCA G and Primer 2

539 (Reverse - TAC ACT ACG TGC CCG CCG AGG) were used to detect SARS-CoV-2 genomes. A

540 primer annealing temperature of $63^{\circ} \mathrm{C}$ was used for all assays.

541 Histological Analysis. The left lung was removed and submerged in $10 \%$ buffered formalin

542 (Fisher) without inflation for 1 week. Lungs from mice sacrificed on day 2 and day 4 were fixed in

543 formalin and paraffin embedded. Five-micron serial sections were taken and used for

544 histopathological staining with hematoxylin and eosin and Immunohistochemistry (IHC) assay.

545 IHC was conducted using rabbit polyclonal antibodies raised against the SARS-CoV nucleocapsid

546 protein (clone NB100-56576 [1:100], Novus Biologicals, Littleton, CO) and biotinylated anti-rabbit

547 IgG, streptavidin AP (Vector Labs, Burlingame, CA) with Permanent Red chromogen

548 (Dako/Agilent, Santa Clara CA). Normal rabbit serum was used as primary antibody for the

549 negative control. Briefly, the sections were deparaffinized by immersion in three xylene baths for

5505 minutes each. The slides were rehydrated by immersion in a series of alcohol baths ranging

551 from 100 to $95 \%$ for 5 minutes each. The slides were pretreated with a citrate-based buffer, pH

5526.0 at $98{ }^{\circ} \mathrm{C}$ for heat-induced epitope retrieval. Endogenous avidin and biotin sites were blocked

553 using avidin/biotin blocking kit (Abcam, Cambridge, MA). The slides were incubated with anti-

554 SARS-CoV antibody for one hour at room temperature, washed in 1X Tris-buffered saline (TBS),

$5550.05 \%$ Tween 20 (wash buffer), and incubated with goat biotinylated anti-rabbit $\lg G(H+L)$ 
antibody for $30 \mathrm{~min}$ at room temperature followed by rinse in wash buffer. Slides were then

557 incubated with streptavidin-AP conjugate for $30 \mathrm{~min}$ at room temperature and washed, followed

558 by incubation with Permanent Red for 5 minutes. Counterstaining with Mayer's hematoxylin

559 solution was performed and the slides mounted with coverslip using Permount mounting medium.

560 Data Availability. The raw data that support the findings of this study are available from the 561 corresponding author upon reasonable request. ${ }^{46}$

562 Biological Materials. Recombinant wild-type and mutant SARS-CoV-2 described in this 563 manuscript will be made available through the World Reference Center for Emerging Viruses and 564 Arboviruses (WRCEVA) at UTMB through material transfer agreement. The SARS-CoV-2 B.1.1.7

565 strain was provided by Dr. Natalie Thornburg and colleagues at the Centers for Disease Control 566 and Prevention.

567 Competing interests

$568 \mathrm{XX}, \mathrm{P}-\mathrm{YS}$, and VDM have filed a patent on the reverse genetic system and reporter SARS-CoV-

569 2. Other authors declare no competing interests.

570 Acknowledgements. Research was supported by grants from NIAID of the NIH to (AI153602 571 and 1R21Al145400 to VDM to P-YS; R24Al120942 (WRCEVA) to SCW, P51OD011132, R56 572 Al147623 and U19AI090023 to MSS. AEM is supported by a Clinical and Translational Science 573 Award NRSA (TL1) Training Core (TL1TR001440) from NIH. ALR was supported by an Institute 574 of Human Infection and Immunity at UTMB COVID-19 Research Fund. Research was also 575 supported by STARs Award provided by the University of Texas System to VDM, and trainee 576 funding provided by the McLaughlin Fellowship Fund at UTMB. P-YS was also supported by CDC 577 grant for the Western Gulf Center of Excellence for Vector-Borne Diseases, and awards from the 578 Sealy \& Smith Foundation, Kleberg Foundation, John S. Dunn Foundation, Amon G. Carter 579 Foundation, Gilson Longenbaugh Foundation, and Summerfield Robert Foundation. MSS was 580 also supported by the Emory Executive Vice President for Health Affairs Synergy Fund award, 581 the Pediatric Research Alliance Center for Childhood Infections and Vaccines and Children's 
582 Healthcare of Atlanta, COVID-Catalyst-I ${ }^{3}$ Funds from the Woodruff Health Sciences Center and

583 Emory School of Medicine, Woodruff Health Sciences Center 2020 COVID-19 CURE Award.

\section{Author Contributions}

585 Conceptualization, AM, MV, BAJ, ALR, MSS, XX, P-YS, VDM ; Methodology, AM, MV, BAJ, MED, 586 AV, PV, KD, ALR, DW, MSS, XX, P-YS; Investigation, AM, MV, BAJ, MED, AV, KL, CS, PV, RML, 587 KD, ALR, DW, MSS, XX, PY-S; Resources, JAP, KSP, SCW, KD, ALR, DW, MSS, XX, P-YS, 588 VDM; Data Curation, AM, MV, BAJ, MED, AV, PV, KD, ALR, DW, MSS, XX, PY-S, VDM.; Writing589 Original Draft, AM, VDM; Writing-Review \& Editing, AM, P-YS, VDM; Data Visualization, AM, KD, 590 VDM; Supervision, SCW, ALR, DW, MSS, P-YS, VDM.; Funding Acquisition, SCW, MSS, P-YS, 591 VDM 
594

595

596

597

598

599

600

601

602

603

604

605

606

607

608

609

610

611

612

613

614

615

616

617

618

619

620

621

622

623

624

625

626

627

628

629

630

631

632

633

634

635

636

637

638

639

640

641

642

643

\section{References}

1 Gralinski, L. E. \& Menachery, V. D. Return of the Coronavirus: 2019-nCoV. Viruses 12, doi:10.3390/v12020135 (2020).

2 Dong, E., Du, H. \& Gardner, L. An interactive web-based dashboard to track COVID-19 in real time. Lancet Infect Dis 20, 533-534, doi:10.1016/S1473-3099(20)30120-1 (2020).

3 Zhu, N. et al. A Novel Coronavirus from Patients with Pneumonia in China, 2019. N Engl J Med 382, 727-733, doi:10.1056/NEJMoa2001017 (2020).

$4 \quad$ Huang, C. et al. Clinical features of patients infected with 2019 novel coronavirus in Wuhan, China. Lancet 395, 497-506, doi:10.1016/S0140-6736(20)30183-5 (2020).

$5 \quad$ Gao, Z. et al. A Systematic Review of Asymptomatic Infections with COVID-19. J Microbiol Immunol Infect, doi:10.1016/j.jmii.2020.05.001 (2020).

6 Lakdawala, S. S. \& Menachery, V. D. The search for a COVID-19 animal model. Science 368, 942-943, doi:10.1126/science.abc6141 (2020).

$7 \quad$ Zhou, P. et al. A pneumonia outbreak associated with a new coronavirus of probable bat origin. Nature 579, 270-273, doi:10.1038/s41586-020-2012-7 (2020).

$8 \quad$ McCray, P. B. et al. Lethal Infection of K18-<em>hACE2</em> Mice Infected with Severe Acute Respiratory Syndrome Coronavirus. Journal of virology 81, 813-821, doi:10.1128/jvi.02012-06 (2007).

9 Menachery, V. D. et al. SARS-like WIV1-CoV poised for human emergence. Proceedings of the National Academy of Sciences of the United States of America 113, 3048-3053, doi:10.1073/pnas.1517719113 (2016).

10 Winkler, E. S. et al. SARS-CoV-2 infection of human ACE2-transgenic mice causes severe lung inflammation and impaired function. Nature immunology 21, 1327-1335, doi:10.1038/s41590-020-0778-2 (2020).

11 Sia, S. F. et al. Pathogenesis and transmission of SARS-CoV-2 in golden hamsters. Nature 583, 834-838, doi:10.1038/s41586-020-2342-5 (2020).

12 Xie, X. et al. An Infectious cDNA Clone of SARS-CoV-2. Cell host \& microbe 27, 841848 e843, doi:10.1016/j.chom.2020.04.004 (2020).

13 Frieman, M. et al. Molecular determinants of severe acute respiratory syndrome coronavirus pathogenesis and virulence in young and aged mouse models of human disease. Journal of virology 86, 884-897, doi:10.1128/JVI.05957-11 (2012).

$14 \mathrm{Gu}, \mathrm{H}$. et al. Adaptation of SARS-CoV-2 in BALB/c mice for testing vaccine efficacy. Science 369, 1603-1607, doi:10.1126/science.abc4730 (2020).

15 Leist, S. R. et al. A Mouse-Adapted SARS-CoV-2 Induces Acute Lung Injury and Mortality in Standard Laboratory Mice. Cell 183, 1070-1085.e1012, doi:10.1016/j.cell.2020.09.050 (2020).

16 Menachery, V. D. et al. A SARS-like cluster of circulating bat coronaviruses shows potential for human emergence. Nature medicine 21, 1508-1513, doi:10.1038/nm.3985 (2015).

17 Roberts, A. et al. A mouse-adapted SARS-coronavirus causes disease and mortality in BALB/c mice. PLoS pathogens 3, e5, doi:10.1371/journal.ppat.0030005 (2007).

18 Xie, X. et al. An Infectious cDNA Clone of SARS-CoV-2. Cell host \& microbe 27, 841848.e843, doi:10.1016/j.chom.2020.04.004 (2020).

19 Doria-Rose, N. et al. Antibody Persistence through 6 Months after the Second Dose of mRNA-1273 Vaccine for Covid-19. N Engl J Med, doi:10.1056/NEJMc2103916 (2021).

20 Widge, A. T. et al. Durability of Responses after SARS-CoV-2 mRNA-1273 Vaccination. N Engl J Med 384, 80-82, doi:10.1056/NEJMc2032195 (2021).

21 Roper, R. L. \& Rehm, K. E. SARS vaccines: where are we? Expert Rev Vaccines 8, 887898, doi:10.1586/erv.09.43 (2009). 
64422 Enjuanes, L. et al. Vaccines to prevent severe acute respiratory syndrome coronavirusinduced disease. Virus Res 133, 45-62, doi:10.1016/j.virusres.2007.01.021 (2008).

23 Krammer, F. Correlates of protection from SARS-CoV-2 infection. Lancet 397, 14211423, doi:10.1016/s0140-6736(21)00782-0 (2021).

24 Channappanavar, R., Zhao, J. \& Perlman, S. T cell-mediated immune response to respiratory coronaviruses. Immunol Res 59, 118-128, doi:10.1007/s12026-014-8534-z (2014).

25 Gouma, S. et al. Sero-monitoring of health care workers reveals complex relationships between common coronavirus antibodies and SARS-CoV-2 severity. medRxiv, 2021.2004.2012.21255324, doi:10.1101/2021.04.12.21255324 (2021).

26 Plante, J. A. et al. The variant gambit: COVID-19's next move. Cell host \& microbe 29, 508-515, doi:10.1016/j.chom.2021.02.020 (2021).

657

27 Liu, Y. et al. The N501Y spike substitution enhances SARS-CoV-2 transmission.

658 bioRxiv, doi:10.1101/2021.03.08.434499 (2021).

659

660 Jerry Zhou, Mark Zeller, Nate Matteson, Chunlei Wu, Kristian G. Andersen, Andrew I. $\mathrm{Su}$, Karthik Gangavarapu, Laura D. Hughes, and the Center for Viral Systems Biology. . S: K417N; S:N501Y; S:H655Y; N128Y Mutation Report.

, <https://outbreak.info/situation-reports?pango\&muts=S\%3A\%20K417N). > (2021).

29 Elbe, S. \& Buckland-Merrett, G. Data, disease and diplomacy: GISAID's innovative contribution to global health. Global challenges (Hoboken, $N J)$ 1, 33-46, doi:10.1002/gch2.1018 (2017).

$30 \mathrm{Li}, \mathrm{F}$. Receptor recognition mechanisms of coronaviruses: a decade of structural studies. Journal of virology 89, 1954-1964, doi:10.1128/JVI.02615-14 (2015).

31 Harcourt, J. et al. Severe Acute Respiratory Syndrome Coronavirus 2 from Patient with 2019 Novel Coronavirus Disease, United States. Emerg Infect Dis 26, doi:10.3201/eid2606.200516 (2020).

32 Sims, A. C. et al. Release of severe acute respiratory syndrome coronavirus nuclear import block enhances host transcription in human lung cells. J Virol 87, 3885-3902, doi:10.1128/JVI.02520-12 (2013).

33 Josset, L. et al. Cell host response to infection with novel human coronavirus EMC predicts potential antivirals and important differences with SARS coronavirus. MBio 4, e00165-00113, doi:10.1128/mBio.00165-13 (2013).

34 Waterhouse, A. et al. SWISS-MODEL: homology modelling of protein structures and complexes. Nucleic Acids Res 46, W296-W303, doi:10.1093/nar/gky427 (2018).

35 Bienert, S. et al. The SWISS-MODEL Repository-new features and functionality. Nucleic Acids Res 45, D313-D319, doi:10.1093/nar/gkw1132 (2017).

36 Xie, X. et al. Engineering SARS-CoV-2 using a reverse genetic system. Nat Protoc 16, 1761-1784, doi:10.1038/s41596-021-00491-8 (2021).

37 Vanderheiden, A. et al. Type I and Type III Interferons Restrict SARS-CoV-2 Infection of Human Airway Epithelial Cultures. Journal of virology 94, doi:10.1128/jvi.00985-20 (2020).

38 Routh, A., Head, S. R., Ordoukhanian, P. \& Johnson, J. E. ClickSeq: FragmentationFree Next-Generation Sequencing via Click Ligation of Adaptors to Stochastically Terminated 3'-Azido cDNAs. J Mol Biol 427, 2610-2616, doi:10.1016/j.jmb.2015.06.011 (2015).

39 Jaworski, E. et al. Tiled-ClickSeq for targeted sequencing of complete coronavirus genomes with simultaneous capture of RNA recombination and minority variants.

692 bioRxiv, 2021.2003.2010.434828, doi:10.1101/2021.03.10.434828 (2021). 
69340 Routh, A. \& Johnson, J. E. Discovery of functional genomic motifs in viruses with ViReMa-a Virus Recombination Mapper-for analysis of next-generation sequencing data. Nucleic Acids Res 42, e11, doi:10.1093/nar/gkt916 (2014).

41 Smith, T., Heger, A. \& Sudbery, I. UMI-tools: modeling sequencing errors in Unique Molecular Identifiers to improve quantification accuracy. Genome Res 27, 491-499, doi:10.1101/gr.209601.116 (2017).

42 Walker, B. J. et al. Pilon: an integrated tool for comprehensive microbial variant detection and genome assembly improvement. PloS one 9, e112963, doi:10.1371/journal.pone.0112963 (2014).

$43 \mathrm{Li}, \mathrm{H}$. et al. The Sequence Alignment/Map format and SAMtools. Bioinformatics 25, 2078-2079, doi:10.1093/bioinformatics/btp352 (2009).

44 Muruato, A. E. et al. A high-throughput neutralizing antibody assay for COVID-19 diagnosis and vaccine evaluation. Nature communications 11, 4059, doi:10.1038/s41467-020-17892-0 (2020).

45 Sheahan, T. et al. Successful vaccination strategies that protect aged mice from lethal challenge from influenza virus and heterologous severe acute respiratory syndrome coronavirus. Journal of virology 85, 217-230, doi:10.1128/JVI.01805-10 (2011).

46 Gralinski, L. E. et al. Mechanisms of Severe Acute Respiratory Syndrome CoronavirusInduced Acute Lung Injury. mBio 4, doi:ARTN e00271-13 